International Journal of Computational Fluid Dynamics

Vol. 00, No. 00, Month 2019, 1-33

This is an Accepted Manuscript of an article published by Taylor \& Francis in International Journal of Computational Fluid Dynamics on April 27, 2020, available online: http://www.tandfonline.com/10.1080/10618562.2020.1753712.

\title{
article
}

\section{Comparison of turbulent Prandtl number correction models for the Stanton evaluation over rough surfaces}

\author{
F. Morency ${ }^{a *}$, H. Beaugendre ${ }^{b, c}$ \\ ${ }^{a}$ Department of Mechanical Engineering, École de technologie supérieure, 1100 \\ Notre-Dame Street West, Montreal, QC, Canada, H3C 1K3; \\ ${ }^{b}$ Univ. Bordeaux, CNRS, Bordeaux INP, IMB, UMR 5251, F-33400, Talence, France; \\ ${ }^{c}$ INRIA, IMB, UMR 5251, F-33400, Talence, France.
}

(April 6, 2020)

In-flight ice accretion code predictions depend on heat loss over rough surfaces. The equivalent sand grain roughness models the friction coefficient, but an additional model is needed for heat transfer predictions. In this paper, a two parameters model based on a turbulent Prandtl number correction is derived from the sublayer Stanton-based model. For flow over rough surfaces, the new model predictions will be compared to a three parameters model from the literature. First, the models are coupled with the Spalart-Allmaras turbulence model to predict heat transfers over rough surfaces. Thereafter, the two models are implemented in the open source software, SU2, and then verified and validated for flow over rough flat plates. Lastly, the predictions of the two models are shown to be similar for ice accretion roughness on flat plates, airfoils and a wing. Model discrepancies are always within the experimental $15 \%$ error margin.

Keywords: Turbulence, heat transfer, roughness, icing, turbulent Prandtl model.

\section{Introduction}

Aircraft ice accretion models, such as those used in Lewice (Wright 2008), IGLOO2D (Trontin et al. 2017), or FENSAP-ICE (Beaugendre et al. 2006), are commonly used to increase the safety of aircraft flying in icing conditions. The overall ice shapes depend strongly on the local convective coefficient, which in turn is a function of the surface roughness (Liu and Hu 2018). Early experimental works by Shin (1996) showed that the

${ }^{*}$ Corresponding author. Email: francois.morency@etsmtl.ca 
size and the distribution of the ice roughness depend on the icing parameters used. Anderson and Shin (1997) and Anderson et al. (2003) established a correlation between the ice roughness element diameters and two dimensionless parameters, the accumulation parameter and the freezing fraction. Nevertheless, a common practice in ice accretion code validation is to rely on a single parameter, namely, the equivalent sand grain roughness height (Han and Palacios 2017). However, Hanson and Kinsel (2019) and Aupoix (2015) recently proposed models that use other parameters. The equivalent sand grain roughness increases the friction and the heat transfer coefficients by altering the boundary conditions at the wall. This in turn changes the resulting ice shape, as shown by Beaugendre et al. (2003). The equivalent sand grain roughness height is obtained from empirical correlations, which are sometimes a function of the freezing fraction at the stagnation point (Walker et al. 2014). For Lewice (Wright 2008), these correlations are developed by selecting the equivalent sand grain roughness height that provides the best agreement with a set of selected ice shapes. Ice shapes depend on many models, such as the ice accretion model, the ice growth model and the number of ice layers in the simulation. Current icing codes need improvement in many models to predict the ice shape more accurately. Investigating the heat transfer prediction is one of the essential steps required to enhance agreement with experiment, as concluded by Hanson and Kinsel (2019). RANS simulations could help investigate flow over significant wall roughness, but near wall models need to be improved (Corson et al. 2009).

Owen and Thomson (1963) were among the firsts to propose a model for the heat transfer over rough surfaces. They introduced the sublayer Stanton number concept to model the effect of horseshoe eddies at the bottom of roughness elements on the heat transfer. Using experimental results, they suggested that the sublayer Stanton number should be a function of the roughness Reynolds number and the Prandtl number. Independently, using their own experimental setup, Dipprey and Sabersky (1963) suggested a similar expression for the sublayer Stanton number, but with different constant values. Ligrani et al. (1979) implemented this heat transfer model into a boundary layer code to predict heat transfer over rough surfaces. The inverse of the sublayer Stanton number was used to shift the temperature at the wall. Later, Kays and Crawford (1993) also used the sublayer Stanton number with an integral method to derive a relation to shift the temperature at the wall and compute the heat transfer above a rough surface. These models use the shear velocity over a rough surface to compute the roughness Reynolds number.

The equivalent sand grain roughness height, $h_{s}$, computed from a correlation for known roughness geometries (Dirling 1973, Pailhas et al. 2008), enables the evaluation of the skin friction coefficient. For RANS turbulence models, the equivalent sand grain roughness is used to impose a non-zero turbulent viscosity at the wall (Aupoix and Spalart 2003, Knopp et al. 2009). The extension of the Spalart-Allmaras turbulence model developed by Boeing to take into account the roughness, called SA Rough in this paper, correctly predicts the wall friction coefficient (Aupoix and Spalart 2003). This model assumes that the flow is averaged over numerous roughness elements, such that there is no need to discretize and solve the flow equations around each of the roughness elements. Instead, the roughness effect on the flow is modeled by an equivalent sand grain roughness height. The value of $h_{s}$ is selected such that the shear stress at the wall is equivalent to the one above the real roughness elements. The non-zero turbulent viscosity at the wall increases the eddy diffusivity for the momentum equations, thus resulting in an increase in the friction coefficient. Due to the turbulent Prandtl number assumption, the eddy diffusivity for energy is also higher near the wall, and the heat flux values therefore increase as 
compared to the smooth wall values.

The SA Rough model is sufficient to predict the shear stress at the wall, but additional corrections are needed to model the heat transfer. Suga et al. (2006) suggested increasing the turbulent Prandtl number in the roughness region to account for the thermal barrier created by the fluid trapped at the bottom of the roughness elements. The additional constant expression for their model was obtained through numerical experiments referring to thermal log-law profiles. Instead of increasing the turbulent Prandtl number near the rough wall, Hink et al. (2014) directly increased the turbulent thermal conductivity by a damping function. An additional polynomial damping function was needed in their model, and the coefficients were devised by fitting the heat transfer prediction to available experiments. Aupoix (2015) suggested a correction for the turbulent Prandtl number near the rough wall, together with two additional parameters and three additional expressions, to compute the turbulent Prandtl number correction. He used numerical results from a discrete element approach to calibrate his model. Chedevergne (2018) extended the use of the Aupoix thermal model to an analytical wall function to avoid the need for fine meshes at the wall.

Most ice accretion codes used heat transfer models based on the sublayer Stanton number and an equivalent sand grain roughness height with an integral method (Wright 2008). Kays and Crawford (1993) integral method corrects the turbulent heat transfer prediction through a shift of the effective wall surface temperature. The model needs one parameter, $h_{s}$, although its constants can be adjusted to match the roughness shapes, as proposed by Owen and Thomson (1963). RANS based ice accretion codes, could benefit from a model based on the sublayer Stanton number of Dipprey and Sabersky (1963). It could ease the transition between RANS and integral method based ice accretion codes. The main objective of this paper is to derive and implement a two parameters model, called the two parameters Prandtl correction (2PP correction) based on the thermal correction model of Dipprey and Sabersky (1963) that can compute roughness thermal effects above fully rough surface and transitional roughness. A secondary objective is to compare the results of the $2 \mathrm{PP}$ correction against the results predicted by the relatively new Aupoix (2015) correction.

First, the three parameters model proposed by Aupoix (2015), named Aupoix Prandtl correction in this paper, and the $2 \mathrm{PP}$ correction used to compute the turbulent Prandtl number above a rough wall are presented. Then, the two turbulent Prandtl number correction models are implemented, verified, and validated against the literature. Finally, the differences between the heat transfer predicted with the Aupoix Prandtl correction and the $2 \mathrm{PP}$ correction over a rough wing and iced airfoils in atmospheric conditions typical of aircraft icing are quantified.

In this paper, the two different thermal models are implemented in SU2 5.2, a computational analysis and design package (Economon et al. 2016). The SA Rough turbulence model is first added to the turbulence models available in SU2. Then, the two thermal models are implemented in SU2 to improve the heat transfer prediction. These models assume an increase in the turbulent Prandtl number near the wall in a bid to reduce the heat transfer. In addition to the equivalent sand grain roughness height, these models require the specification of one or two parameters to characterize the roughness shape. 


\section{Thermal roughness models}

In this section, the two turbulent Prandtl correction models are presented and validated. The general equations for the computation of the turbulent heat transfer above a rough wall are defined first. Then, the Aupoix Prandtl correction is exposed, in the context of the RANS variables readily available in SU2. Next, the $2 \mathrm{PP}$ correction model is derived and calibrated against the models of Ligrani et al. (1979).

The thermal roughness models are added to the CFD code in a two-step process. The process begins with the extension of the turbulence model to include the roughness effects on the momentum equations. For the Spalart-Allmaras model, the work of Aupoix and Spalart (2003) suggests several test cases for verification and validation. The experimental results of Hosni et al. (1993) are often exploited for model development. The SU2 implementation of the SA Rough model has been partially validated in Tagawa et al. (2018). In the present paper, additional comparisons are made with the test cases utilized by Knopp et al. (2009). Although the latter paper focuses on the $k-\omega$ model extension, it also presents numerical results obtained with an implementation of the SA turbulence model with a constant Prandtl number. The process ends with the implementation of the thermal correction. For thermal correction validations, the experiments for flows over rough flat plates by Healzer et al. (1974), Ligrani et al. (1979), and Hosni et al. (1993) are the most commonly used. Although useful for model calibration, these experiments are based on spherical roughness elements that are not necessarily representative of ice accretion roughness. The experimental results of Dukhan et al. (1999) are more relevant for icing, and have been used by Radenac et al. (2018) for extended rough wall model validation.

\subsection{Heat transfer above a rough wall}

For a flow over a rough surface, the Figure 1 presents a close-up view of the roughness. The roughness height, $h$, above the wall, is different from the equivalent sand grain roughness height, $h_{s}$. Below a certain height, in the melt-down region, the flow is trapped, and recirculation occurs. For the flow between roughness geometries, the proposed model increases the turbulent Prandtl number, $\mathrm{Pr}_{t}$, and reduces the turbulent heat transfer from the wall. The increase in the turbulent Prandtl number, $\Delta \operatorname{Pr}_{t}$, should be a smooth function of $y$, such that it goes from a maximum value at the wall to the free-stream value, $\operatorname{Pr}_{t}$, above the roughness. In the figure, the effective Prandtl number, $\operatorname{Pr}_{t}^{e f f}$, is drawn as a function of the normal distance from the smooth wall, $y$.

In a RANS flow solver, a modification of either the effective thermal conductivity, $k_{e}$, or the effective turbulent Prandtl number, $\operatorname{Pr}_{t}^{e f f}$, can model the heat flux reduction caused by the flow trapped at the bottom of the roughness elements. The heat flux at the wall, $q_{w}$, depends on the temperature gradient in the normal direction and on $k_{e}$ :

$$
q_{w}=-k_{e}\left(\frac{\partial T}{\partial n}\right)_{w}
$$

The Stanton number, a dimensionless number that characterizes the heat transfer from the wall to the fluid, is defined as 


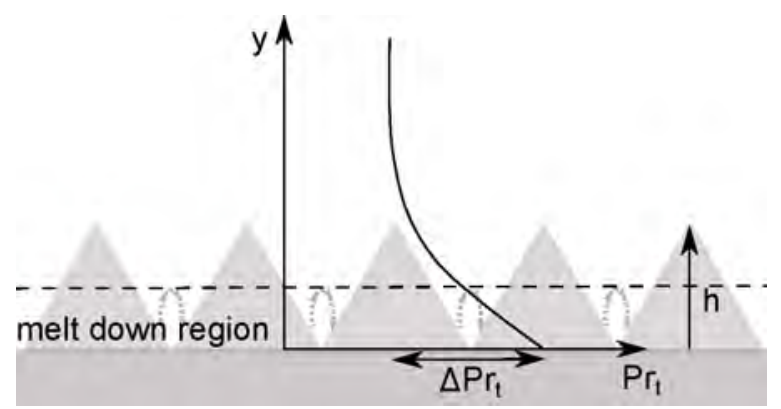

Figure 1. Turbulent Prandtl number evolution above a rough wall

$$
\mathrm{St}=\frac{q_{w}}{\rho U_{\infty} c_{p}\left(T_{t}-T_{w}\right)}
$$

with $U_{\infty}$ the free stream velocity, $c_{p}$ the specific heat, $T_{w}$ the wall temperature and $T_{t}$ the total temperature.

For turbulent flows, $k_{e}$ is related to the laminar thermal conductivity, $k_{L}$, and the turbulent thermal conductivity, $k_{t}$ :

$$
\begin{aligned}
& k_{e}=k_{L}+k_{t} \\
& k_{L}=\frac{c_{p} \mu_{L}}{\operatorname{Pr}} \\
& k_{t}=\frac{c_{p} \mu_{t}}{\operatorname{Pr}_{t}^{e f f}}
\end{aligned}
$$

where $\mu_{L}$ is the laminar viscosity, and $\mu_{t}$ is the turbulent viscosity.

By definition, an increase in $\operatorname{Pr}_{t}^{e f f}$ is equivalent to a reduction of $k_{t}$. Calvo (2009) suggests limiting the thermal eddy conductivity $k_{t}$ by a damping function, which depends on the dimensionless wall normal distance, the $\operatorname{Pr}_{t}$, and the $\operatorname{Re}_{s}$. Equivalently, the $\operatorname{Pr}_{t}$ can be corrected near the rough wall by a given value, $\Delta \operatorname{Pr}_{t}$ which is a function of the rough wall distance and the roughness element shape:

$$
\operatorname{Pr}_{t}^{e f f}=\operatorname{Pr}_{t}+\Delta \operatorname{Pr}_{t}
$$

For a turbulent flow over a smooth wall, the usual assumptions are that $\operatorname{Pr}_{t}=0.9$ everywhere and $\mu_{t}=0$ at the wall. However, $\mu_{t}>0$ in the case of the SA Rough model, see appendix A. It is important to note that the $\Delta \operatorname{Pr}_{t}$ function does not change the SA Rough model. Thus, the $\Delta \operatorname{Pr}_{t}$ values are computed in the post-processing step at each iteration, after the turbulence model equation is solved. 


\subsection{Aupoix Prandtl correction}

Aupoix (2015) derived a model for the $\Delta \operatorname{Pr}_{t}$ based on numerical results obtained with the discrete element approach. This approach is able to reproduce the difference between drag and heat transfer enhancement by surface roughness. The discrete element approach is formulated for the boundary layer equations and has been thoroughly validated. Aupoix (2015) uses it as an alternative to DNS simulations or experiments for the calibration of the following correction function:

$$
\Delta \operatorname{Pr}_{t}=F\left(\Delta u^{+}, S_{\text {corr }}\right) G\left(\frac{y}{h}\right)
$$

where $S_{\text {corr }}$ is the corrected wetted surface ratio, and $\Delta u^{+}$is the shift of the mean velocity profile. Nikuradse related the shift $\Delta u^{+}$to the roughness Reynolds number, $\mathrm{Re}_{s}$, as mentioned in Aupoix (2007). The $\operatorname{Re}_{s}$ is a function of the shear velocity at the wall, $u_{\tau}$, and $h_{s}$

$$
\operatorname{Re}_{s}=\frac{u_{\tau} h_{s}}{\nu}
$$

An alternative relation, derived by Grigson can also be used, according to Radenac et al. (2018)

$$
\Delta u^{+}=\frac{1}{\kappa} \ln \left(1+\frac{\operatorname{Re}_{s}}{e^{1.3325}}\right)
$$

where $\kappa=0.41$.

The function $G$ is designed to restrict the $\Delta \operatorname{Pr}_{t}$ correction to the roughness sublayer, because experimental measurements in the boundary layer above rough surfaces show that $\operatorname{Pr}_{t}$ values are still around 0.9:

$$
G\left(\frac{y}{h}\right)=\exp \left(-\frac{y}{h}\right)
$$

where $y$ is the normal distance to the closest wall. The function, $F$, depends on the ratio of the corrected rough wetted surface to the smooth surface, $S_{\text {corr }}$. The corrected wetted surface calculation neglects the troughs below the melt-down surface, as defined in the discrete element approach (Aupoix 2015). This ratio varies with the shape of the single roughness element and the ratio of the roughness spacing over the roughness height. The typical values are between 1, for large roughness spacing, and 1.4, for densely packed cones:

$$
\begin{aligned}
F\left(\Delta u^{+}, S_{\text {corr }}\right) & =A \Delta u^{+2}+B \Delta u^{+} \\
A & =\left(0.0155-0.0035 S_{\text {corr }}\right)\left(1.0-\exp \left(-12 .\left(S_{\text {corr }}-1.0\right)\right)\right) \\
B & =-0.08+0.25 \exp \left(-10 .\left(S_{\text {corr }}-1.0\right)\right)
\end{aligned}
$$


The shear velocity $u_{\tau}$ is needed to compute $\operatorname{Re}_{s}$. This velocity is not readily available at node points above the wall in SU2. However, the Spalart-Allmaras turbulence model has been tuned such that the $\tilde{\nu}$ value is related to $u_{\tau}$ and the corrected rough wall distance $d+0.03 h_{s}$ (Aupoix and Spalart 2003):

$$
u_{\tau}=\frac{\tilde{\nu}}{\kappa\left(d+0.03 h_{s}\right)}
$$

According to Calvo (2009), this solution applies in the fully rough area, but leads to poorer predictions in the transitionally rough area, when $0.03 h_{s}$ does no longer hold. Nevertheless, this approximation is used in this paper, and gives good predictions. In the SU2 model implementation, a modification of the original Aupoix model prevents the $F$ function from taking negative values in the course of the iterative solution, $F=$ $\max (0, F)$. Negative $F$ values can occur when $\Delta u^{+}$values are near zero, depending on the values of $A$ and $B$.

\subsection{Two parameters Prandtl correction (2PP correction)}

A two parameters model to compute the $\Delta \mathrm{Pr}_{t}$ is derived based on the works of Ligrani et al. (1979) and Dipprey and Sabersky (1963). Experiments at Stanford led to a model based on an increase in the rough wall temperature by a value $\delta T_{0}$ (Ligrani et al. 1979). This temperature shift is usually normalized by the friction temperature $T_{\tau}$

$$
\begin{aligned}
T_{\tau} & =\frac{-q_{w}}{\rho c_{p} u_{\tau}} \\
\delta T_{0}^{+} & =\frac{\delta T_{0} \rho c_{p} u_{\tau}}{q_{w}}
\end{aligned}
$$

This model is coupled with an integral method for heat transfer prediction over rough surfaces in the advanced heat transfer work of Kays and Crawford (1993). Over a rough surface, the temperature $T^{+}$is obtained by integration:

$$
\begin{aligned}
T^{+}=\frac{\left(T_{w}-T\right) u_{\tau}}{q_{w} /\left(\rho c_{p}\right)} & =\int_{0}^{y^{+}} \frac{\mathrm{d} y^{+}}{1 / \operatorname{Pr}+k_{t} /\left(\rho c_{p} \nu\right)} \\
& \approx \int_{0}^{y_{v}^{+}} \operatorname{Prd} y^{+}+\int_{y_{v}^{+}}^{y^{+}} \frac{\mathrm{d} y^{+}}{k_{t} /\left(\rho c_{p} \nu\right)}
\end{aligned}
$$

where $y_{v}^{+}$is the effective conduction sublayer thickness, $y_{v} u_{\tau} / \nu$. In the logarithmic region of a rough boundary layer, we have, according to Kays and Crawford (1993):

$$
\frac{\nu_{t}}{\nu}=\kappa\left(y^{+}+\delta y_{0}^{+}\right)
$$

with $y^{+}=y u_{\tau} / \nu$ and $\delta y_{0}^{+}=0.031 \operatorname{Re}_{s}$. With $\operatorname{Pr}_{t}$ defined as $k_{t} / \rho c_{p} \nu$, the second integral on the right hand side of the equation (18) becomes: 


$$
\int_{y_{v}^{+}}^{y^{+}} \frac{\mathrm{d} y^{+}}{k_{t} /\left(\rho c_{p} \nu\right)}=\int_{y_{v}^{+}}^{y^{+}} \frac{\operatorname{Pr}_{t} \mathrm{~d} y^{+}}{\kappa\left(y^{+}+\delta y_{0}^{+}\right)}
$$

After integration, equation (20) is:

$$
\int_{y_{v}^{+}}^{y^{+}} \frac{\operatorname{Pr}_{t} \mathrm{~d} y^{+}}{\kappa\left(y^{+}+\delta y_{0}^{+}\right)}=\frac{\operatorname{Pr}_{t}}{\kappa} \ln \left(\frac{y^{+}+\delta y_{0}^{+}}{\delta y_{0}^{+}+y_{v}^{+}}\right)
$$

If $y_{v}^{+}<<\delta y_{0}^{+}$, the temperature $T^{+}$is:

$$
T^{+}=\frac{\operatorname{Pr}_{t}}{\kappa} \ln \left(\frac{y^{+}+\delta y_{0}^{+}}{\delta y_{0}^{+}}\right)+\int_{0}^{y_{v}^{+}} \operatorname{Pr} \mathrm{d} y^{+}
$$

If we make the assumption that only molecular effects, $\mathrm{Pr}$, account for the thermal transport in the effective conduction sublayer, from 0 to $y_{v}^{+}$, then

$$
T^{+}=\frac{\operatorname{Pr}_{t}}{\kappa} \ln \left(\frac{y^{+}+\delta y_{0}^{+}}{\delta y_{0}^{+}}\right)+\delta T_{0}^{+}
$$

The average conduction sublayer thickness $y_{v}^{+}$depends on the roughness geometry, the flow velocity, and the fluid properties. Dipprey and Sabersky (1963) and Owen and Thomson (1963) found that experimental data could be represented by

$$
\delta T_{0}^{+}=\frac{\operatorname{Re}_{s}^{\alpha} \operatorname{Pr}^{\beta}}{C}
$$

Experiments over flat plates covered with roughness provide the values for $C, \alpha$, and $\beta$.

Equation (24) is used with values $C=1.92, \alpha=0.45$, and $\beta=0.8$ in most icing codes, according to Radenac et al. (2018). Kays and Crawford (1993) suggest instead $C=0.8$, $\alpha=0.2$, and $\beta=0.44$. The constant $C$ depends on the roughness geometry. Ligrani et al. (1979) suggest multiplying equation (24) by a function $g$ to reduce the temperature step $\delta T_{0}^{+}$when the roughness Reynolds number is in the transitionally rough regime. As suggested by Kays and Crawford (1993), the smooth regime occurs if $\operatorname{Re}_{s}<5$, and the fully rough regime if $\operatorname{Re}_{s}>70$. This leads to the following equation:

$$
\begin{aligned}
\delta T_{0}^{+} & =g \frac{\operatorname{Re}_{s}^{\alpha} \operatorname{Pr}^{\beta}}{C} \\
g & =1 \text { if } \operatorname{Re}_{s} \geq 70 \\
g & =\frac{\ln \operatorname{Re}_{s}-\ln 5}{\ln 70-\ln 5} \text { if } 5<R e_{s}<70 \\
g & =0 \text { if } \operatorname{Re}_{s} \leq 5
\end{aligned}
$$


As the function $g$ only corrects the thermal model, the smooth wall behavior for $\operatorname{Re}_{s} \leq 5$ is only recovered as long as the momentum model, the SA Rough model in our case, captures it.

The equation (25) is shown to be equivalent to the corrected turbulent Prandtl, $\Delta \operatorname{Pr}_{t}$. If we replace the $\operatorname{Pr}_{t}$ in equation (20) with $\operatorname{Pr}_{t}^{\text {eff }}=\operatorname{Pr}_{t}+\Delta \operatorname{Pr}_{t}$, the integral is:

$$
T^{+}=\int_{y_{v}^{+}}^{y^{+}} \frac{\operatorname{Pr}_{t} \mathrm{~d} y^{+}}{\kappa\left(y^{+}+\delta y_{0}^{+}\right)}+\int_{y_{v}^{+}}^{y^{+}} \frac{\Delta \operatorname{Pr}_{t} \mathrm{~d} y^{+}}{\kappa\left(y^{+}+\delta y_{0}^{+}\right)}
$$

By comparison with equations (18) and (23), the last term on the right hand side is equivalent to $\delta T_{0}^{+}$, if $y_{v}^{+}<<\delta y_{0}^{+}$. Let us assume that the corrected turbulent Prandtl takes the form $\Delta \operatorname{Pr}_{t}=F \exp (-y / h)$, similar to equation (7). If $h=h_{s}$, then:

$$
\begin{aligned}
& \delta T_{0}^{+}=\frac{1}{\kappa} \int_{0}^{y^{+}} \frac{F \exp \left(-y^{+} / \operatorname{Re}_{s}\right) \mathrm{d} y^{+}}{y^{+}+\delta y_{0}^{+}} \\
& \delta T_{0}^{+}=\frac{1}{\kappa} F \int_{0}^{y^{+}} \frac{\exp \left(-y^{+} / \operatorname{Re}_{s}\right) \mathrm{d} y^{+}}{y^{+}+\delta y_{0}^{+}}
\end{aligned}
$$

To evaluate the value of the constant $F$, the following assumptions are made: $\delta y_{0}^{+}=$ $0.031 \mathrm{Re}_{s}$. The upper integral bound value was selected by trial and error, such that a further increase in the value does not change the integration results. If the integral bounds are from 0 to $y^{+}=10 \operatorname{Re}_{s}$, the integral in equation (31) reaches an almost constant numerical value:

$$
\begin{aligned}
\delta T_{0}^{+} & =\frac{1}{\kappa} F \int_{0}^{10 \operatorname{Re}_{s}} \frac{\exp \left(-y^{+} / \mathrm{Re}_{s}\right) \mathrm{d} y^{+}}{y^{+}+0.031 \mathrm{Re}_{s}} \\
\delta T_{0}^{+} & \approx \frac{1}{\kappa} F 3.02
\end{aligned}
$$

Then, the value $F$ is a function of $\operatorname{Re}_{s}$ and $\operatorname{Pr}$ :

$$
F=\frac{\kappa}{3.02} \frac{g \operatorname{Re}_{s}^{\alpha} \operatorname{Pr}^{\beta}}{C}=0.136 \frac{g \operatorname{Re}_{s}^{\alpha} \operatorname{Pr}^{\beta}}{C}
$$

Thus, a $\Delta \operatorname{Pr}_{t}$ function consistent with the semi-empirical correlation used in existing icing codes can be obtained. Radenac et al. (2018) followed similar steps as above to cast the Aupoix thermal model in a form compatible with the integral boundary layer method. In SU2, the correction equation (7) is implemented as $\Delta P r_{t}=F \times G$. The $G$ value is computed with equation (10). The $F$ function is computed either with the equation (11) for the Aupoix Prandtl correction, or with the equation (34) for the $2 \mathrm{PP}$ correction. As the equation (34) relies on $R e_{S}$, the shear velocity $u_{\tau}$ is computed with equation 14. Although the constant values of equation (34) can be modified, the default values $C=1.92, \alpha=0.45$, and $\beta=0.8$ are used for the test cases. 


\subsection{Validation}

The implementation of the SA Rough model is first verified by comparing friction coefficients with those obtained in the literature. Then, the Aupoix Prandtl correction is validated, along with the $2 \mathrm{PP}$ correction against experimental results for flow above rough flat plates.

\subsubsection{Verification of the implementation of the SA Rough model}

The equations for the SA Rough model, as implemented in the CFD code, are detailed in the appendix A, together with the RANS equations. The numerical methods used to solve the RANS equations are detailed in Economon et al. (2016). The equations are discretized with a standard edge-based structure on a dual grid with control volumes constructed using a median-dual vertex-based scheme. Median-dual control volumes are formed by connecting the centroids, face, and edge midpoints of all cells sharing the particular node. The convective and viscous fluxes are evaluated at the midpoint of an edge. The JST scheme, a central scheme with artificial dissipation, is used to compute the convective flux with a Green-Gauss numerical method for the spatial gradients (Blazek 2015). The system of linearized equations is iteratively solved using an implicit first order Euler time-stepping scheme and the GMRES algorithm. The numerical results obtained are compared to numerical results from Aupoix and Spalart (2003) and Knopp et al. (2009). The friction coefficients are verified against the so-called MSU2 test case and the NACA $65_{2} 215$ airfoil results of Knopp et al. For all the test cases in this paper, the flow is assumed to be fully turbulent.

The MSU2 case represents a turbulent boundary layer flow over a rough surface of length $L=2.4$. The farfield velocity is $58 \mathrm{~m} / \mathrm{s}$ and hemispheres of diameter $1.27 \mathrm{~mm}$ create the wall roughness. The equivalent sand grain roughness, $h_{s}=1.0946 \times 10^{-3} \mathrm{~m}$, is computed from the Dirling (1973) correlation equations. The computational domain is similar to the one used for the flat plate turbulent test case available on the NASA turbulence modeling resource webpage (https://turbmodels.larc.nasa.gov/flatplate. html) (Rumsey 2014), but the flat plate length is $5 \mathrm{~m}$ instead of $2 \mathrm{~m}$. The Reynolds number is $1.92 \times 10^{7}$ (based on the flat plate length) and the Mach number is 0.169 . The computational mesh has 98 elements in the normal $y$-direction and 180 elements along the $x$-direction, with 154 elements belonging to the plate. The Jameson-Schmidt-Turkel (JST) numerical scheme is used for the flow inviscid terms in SU2, as it was used for the calculation by Knopp et al. The maximum value for $y^{+}$is 0.5 at the beginning of the plate, and this value falls below 0.3 for $x$ values greater than $0.85 \mathrm{~m}$.

In Figure 2, the friction coefficient computed respectively by SU2, Knopp et al., and Aupoix and Spalart for the MSU2 test case are compared. The three results are obtained with the SA Rough model. Results from the MSU2 experiment are also shown as a reference. As expected, the SU2 and Knopp et al. results are almost identical, even if the meshes were different. While the Aupoix and Spalart results predict a slightly higher friction coefficient value, the model implementation was, however, done in a boundary layer code with numerical methods different from those used in RANS codes.

The model implementation is also verified for a pressure gradient flow, the flow around the NACA $65_{2} 215$ airfoil. The roughness covers the entire upper surface and the first $15 \%$ of the lower airfoil surface near the leading edge. The equivalent sand grain roughness is $h_{s}=3.08 \times 10^{-4} \mathrm{~mm}$ for an airfoil chord of $c=1 \mathrm{~m}$. The Reynold number is $R e=$ $2.6 \times 10^{6}$, the Mach number is $M a=0.182$, and the angle of attack is $12^{\circ}$.

A C-grid computational domain is used around the airfoil, shown on the left of Figure 3, extending 50 chords away from the airfoil leading edge. The CFD results are obtained on 


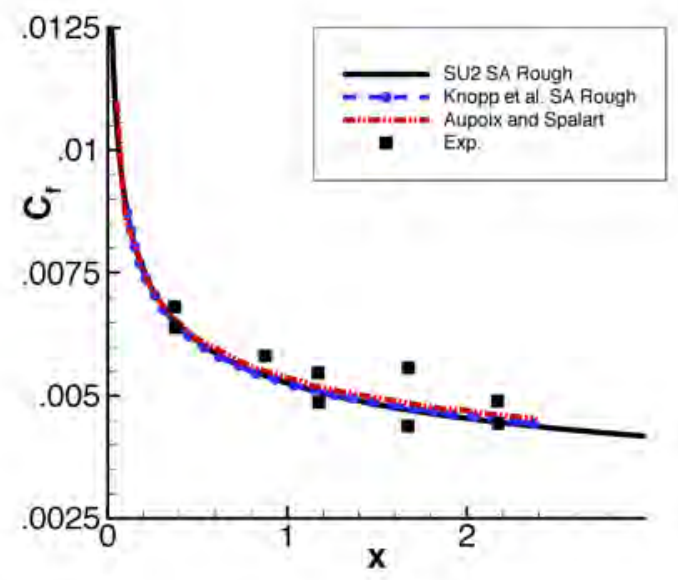

Figure 2. Friction coefficient for the MSU2 case with the SA Rough model implemented in SU2 compared to numerical results from Knopp et al. (2009) and Aupoix and Spalart (2003), and experimental results from Hosni et al. (1993)

three quadrilateral grids: coarse, medium, and fine. The medium mesh has 292 elements on the airfoil boundary and 884 elements on the farfield boundary. The growth ratio between the grids is 1.41 in each direction. Hence, the coarse grid and the fine grid have 206 and 413 elements on the airfoil surface, respectively. The element size in the normal direction is smaller near the airfoil surface, with a spacing between the first node and the surface of $\approx 2 \times 10^{-6} \mathrm{~m}$ and a growth ratio of 1.1 . This gives a $y^{+} \approx 0.2$ on the leading edge area on the upper side of the airfoil for the medium grid.

The computed 2D flow around the airfoil is illustrated on the right of Figure 3. The pressure coefficient iso-contours show a strong pressure gradient on the upper side of the airfoil, with a minimum value of around -7 near the leading edge that goes back to -2 for about $10 \%$ of the chord. The streamlines highlight the separation region near the airfoil trailing edge.

Figure 4 compares the friction coefficients computed by SU2 to that of Knopp et al. (2009) obtained with the SA Rough model. The SU2 friction coefficients are almost the same for the three meshes. The largest discrepancies between SU2 results are near $x / c=0.5$ and near $x / c=0.9$. SU2 predicts the separation point near $x / c=0.75$ on the upper side of the airfoil, while it occurs at $x / c=0.65$ for Knopp et al. Although not presented here, our numerical tests show that the separation point is sensitive to the choice of numerical scheme in SU2. As it is the case in the literature, only the results obtained with the JST scheme are shown. This difference in the separation point location creates differences in the pressure coefficient distribution on the upper side of the airfoil. Therefore, the SU2 friction coefficient is shifted slightly from $x / c=0.3$ to the trailing edge. On the lower side of the airfoil, the friction coefficient changes abruptly at $x / c=0.15$ because the surface goes from rough to smooth. The friction coefficients predicted by SU2 are lower than those obtained in the literature between $x / c=0.1$ and $x / c=0.15$, with a maximum value of $c_{f}=0.0026$, as compared to $c_{f}=0.0028$ for Knopp et al. It should be noted that Knopp et al. used a hybrid mesh, with prismatic 

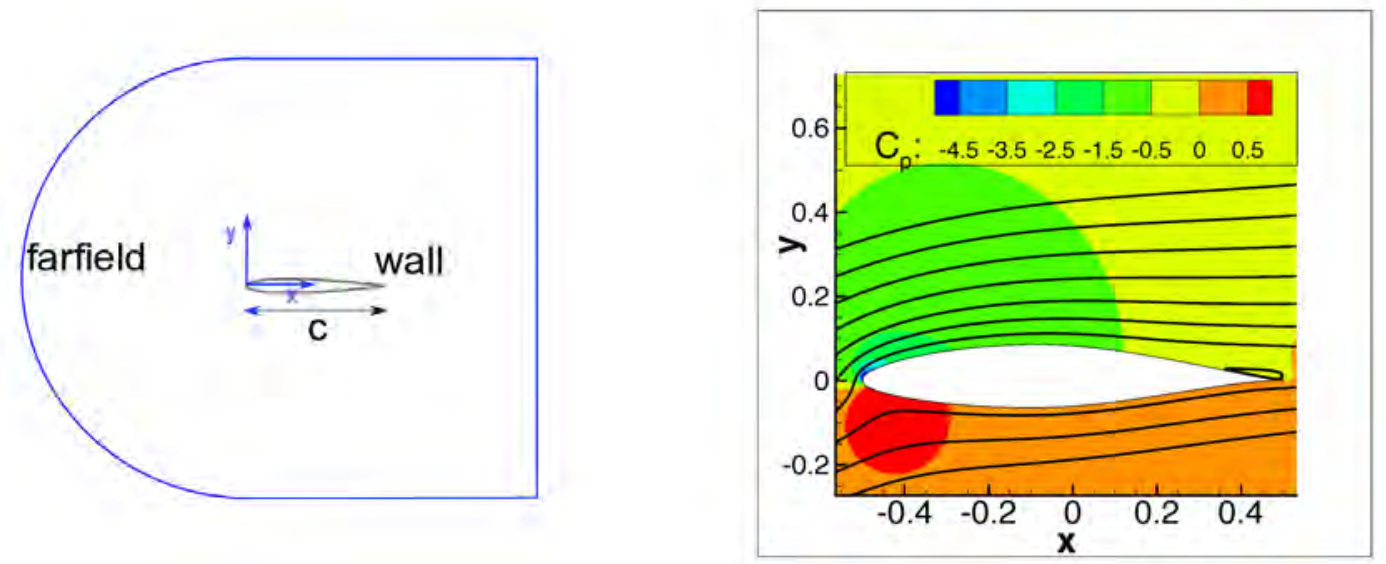

Figure 3. NACA $65_{2} 215$ airfoil at $12^{\circ}$ angle of attack: on the left computational domain out of scale, on the right pressure coefficient distribution and streamlines.

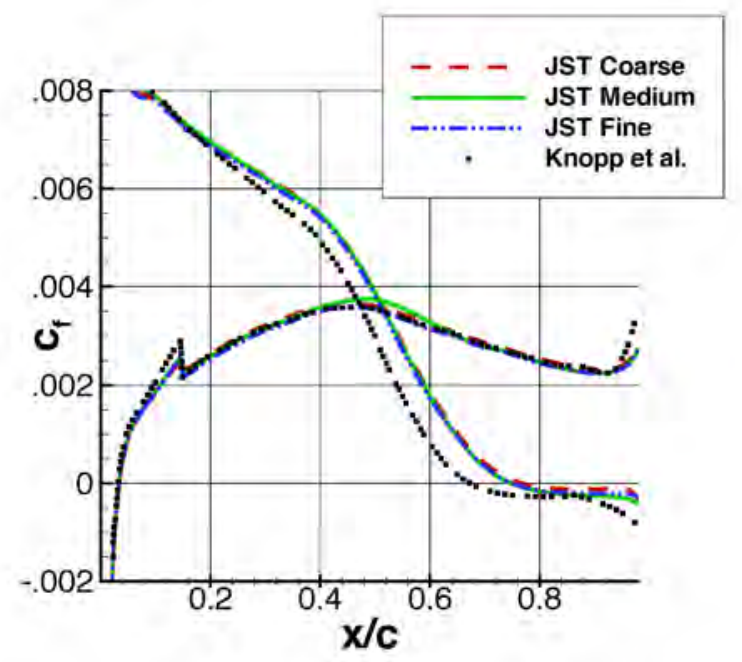

Figure 4. Friction coefficient around the NACA $65_{2} 215$ airfoil at $12^{\circ}$ angle of attack.

elements near the airfoil, triangular elements in the farfield, and an unknown size for the computational domain. Taking this into account, as well as the fact that the exact JST scheme implementation used by Knopp et al. is not known, the comparison between SU2 results and those from literature results is considered satisfactory.

\subsubsection{Validation of Aupoix Prandtl correction (Aupoix 2015)}

The first test case selected to validate the Aupoix Prandtl correction, inside SU2,

corresponds to experiments from Stanford University on flows over a flat plate entirely

scale, on the right pressure coeficient distribution and streamlines.

Figure 4. Fiction coeficient around the NACA 652215 airfoil at $12^{\circ}$ angle of attack.

(1)
if 
covered with closely packed spheres. The spheres had a diameter of $0.635 \mathrm{~mm}$, and the equivalent sand grain roughness was 1.25 times the diameter, i.e., $h_{s}=0.79375 \mathrm{~mm}$. The roughness height is not defined in Aupoix's paper, and half of the sphere diameter, i.e., $h=0.3175 \mathrm{~mm}$, is used in our simulations. The corrected wetted area ratio considered is $S_{\text {corr }}=1.1418$. In the experiments of Healzer et al. (1974), the Stanton numbers are deduced from a thermal balance on heated flat plates. In our numerical simulations, the free-stream temperature is set to $293 \mathrm{~K}$ and the flat plate is heated to $300 \mathrm{~K}$. This is slightly different from the experiments, but St results are not sensitive to this choice. The free-stream velocity is set to $73.8 \mathrm{~m} . \mathrm{s}^{-1}$. In Aupoix's article, the author mentions that the flow is turbulent from the beginning of the measurement region. In our numerical set-up we run the flat plate fully turbulent, and the Reynolds number is set to $4.86 \times 10^{6}$. The corresponding roughness Reynolds number $R e_{s}$ is about 200 for this test case.

First, a mesh sensitivity study was conducted (Figure 5). Three meshes were used: i) coarse mesh; $50 \times 77, \max y^{+}=1.95$, ii) medium mesh; $100 \times 144$, max $y^{+}=1.19$, and iii) fine mesh; $200 \times 288, \max y^{+}=0.89$. We observe the mesh convergence for the friction coefficient and the Stanton number. The Stanton number is more sensitive to the mesh size, as the coarse mesh results are significantly higher than those for the medium and fine meshes.

The results obtained on the fine mesh are then compared to the literature results. In the Figure 6, two SU2 computation results are shown: a first one with the SA Rough model, and a second one with the Aupoix Prandtl correction. The Aupoix Prandtl correction models have almost no effect on the friction coefficient because the temperature difference of $7 \mathrm{~K}$ between the flat plate and the free-stream is too small to induce significant changes in the air properties. As expected, the predicted Stanton numbers are significantly lower when the Aupoix Prandtl correction is used. Both friction coefficients and Stanton numbers are in good agreements with experimental and other Aupoix numerical solutions. The SU2 values are higher than Aupoix's numerical results, but are closer to the experimental results. The Aupoix numerical solutions are obtained with the Clicet code (Aupoix 2010), a boundary layer code. Small discrepancies exist between the Aupoix numerical solution and SU2 near $x=0.5 \mathrm{~m}$. We believe that the different model assumptions at the beginning of the turbulent boundary layer calculations are at the root of these differences.

The second test case validate the model prediction above ice roughness. Dukhan et al. investigated several surfaces corresponding to different icing conditions. The experimental set-up was a flat plate with a $0.0508 \mathrm{~m}$ smooth part before the rough part. The smooth part is adiabatic, while the rough part is heated. Our numerical set-up prescribed a freestream temperature of $293 \mathrm{~K}$ and a heated temperature of $300 \mathrm{~K}$. In our simulations, the laminar/turbulent transition is not prescribed, and the following three test cases are run fully turbulent. The free-stream velocity is set to $47.24 \mathrm{~m} . \mathrm{s}^{-1}$. Three sparse roughness cases are presented here.

The first one, an intermediately spaced rough glaze, corresponds to an equivalent sand grain roughness of $h_{s}=15.63 \mathrm{~mm}$, a corrected wetted area ratio of $S_{\text {corr }}=1.22$, and a roughness height of $h=3.07 \mathrm{~mm}$. The roughness Reynolds number $R e_{s}$, around 4000, is very large for this test case. Here, the Stanton number SA Rough is highly overestimated (see the top of Figure 7). The equivalent sand grain roughness approach, coupled with a constant turbulent Prandtl number model, over predicts the effect of roughness on heat transfer, as previously reported Aupoix and Spalart (2003). Aupoix Prandtl correction significantly improves the predictions as compared to experimental data from Dukhan. The results obtained using SU2 with and without Prandtl correction are in good agree- 

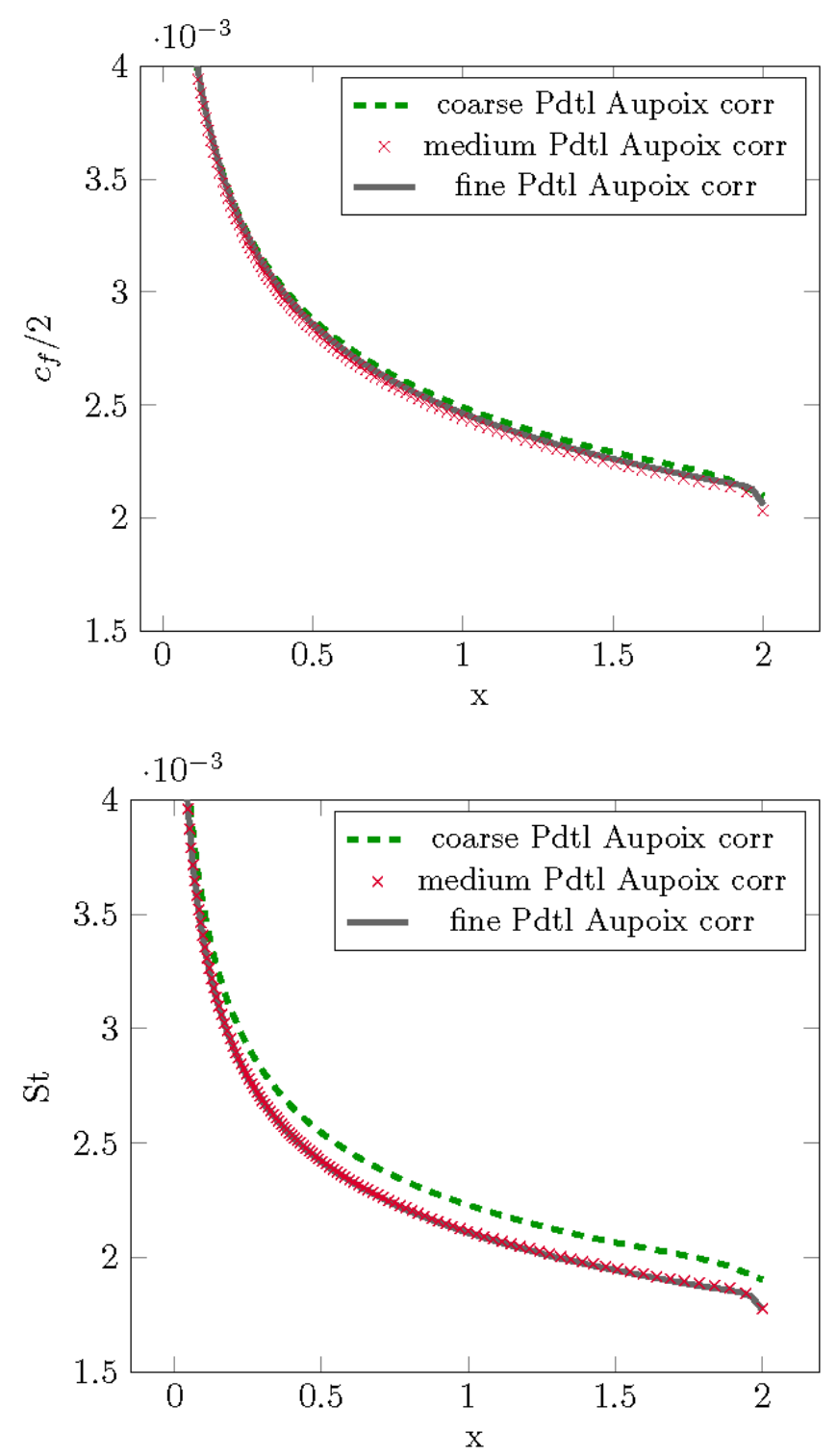

Figure 5. Healzer et al. experiments, mesh sensitivity study.

ment with Aupoix's results (Aupoix 2015).

The second test case, corresponding to a rime feathers condition, is set using an equivalent sand grain roughness of $h_{s}=23.04 \mathrm{~mm}$, a corrected wetted area ratio of $S_{\text {corr }}=1.22$, and a roughness height of $h=4.32 \mathrm{~mm}$. The reduced value of $R e_{s}$ is about 6000 for this case. Again, the numerical results obtained using SU2 are in agreement with the literature and experimental data (see middle of Figure 7). The turbulent Prandtl number correction significantly improves the numerical prediction of the Stanton number. The Stanton number is almost divided by a factor of 2 .

The third test case corresponds to a smooth rime icing condition. The equivalent sand 

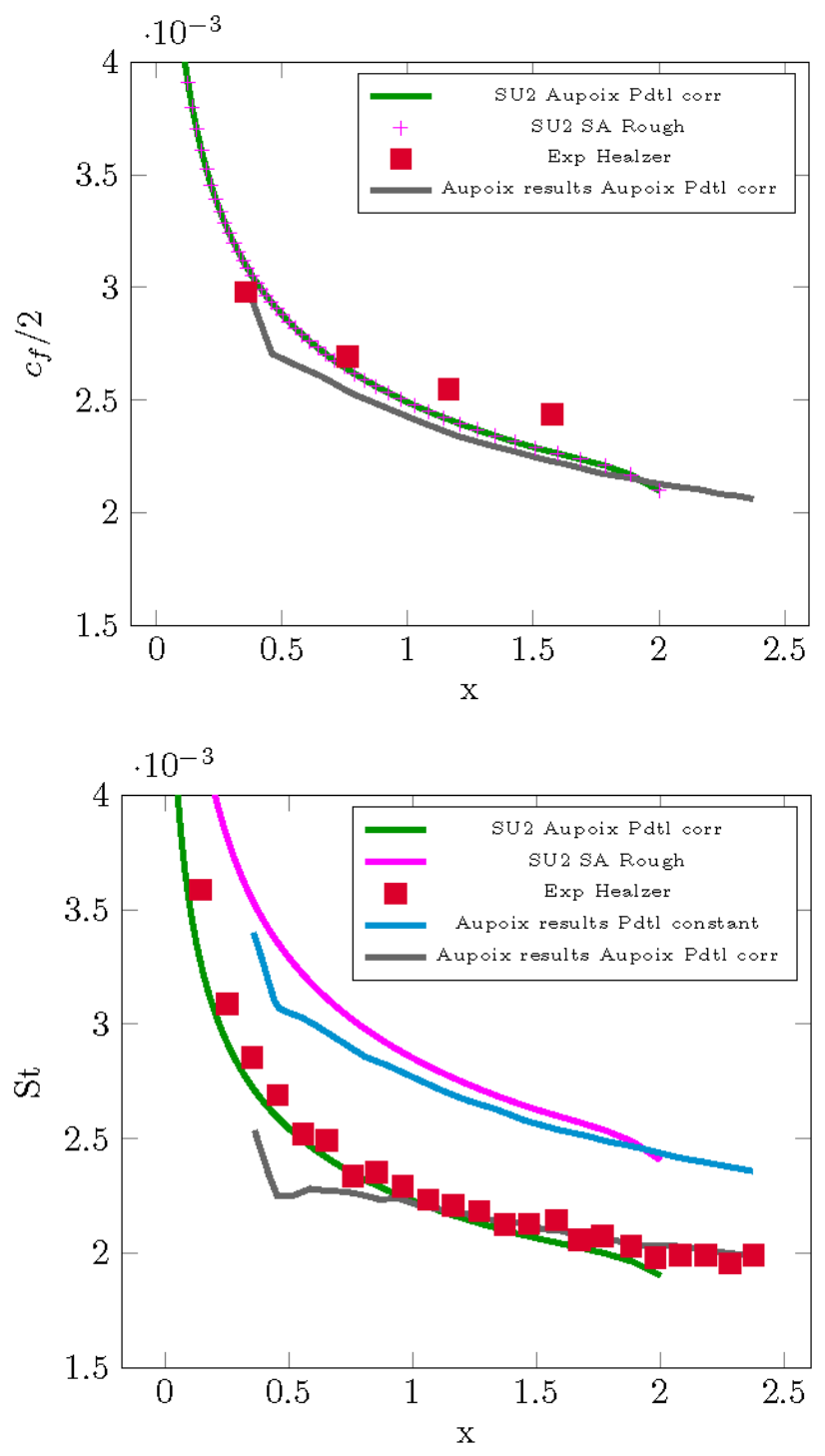

Figure 6. Healzer et al. experiments (Aupoix 2015), most closely packed spheres: top - skin friction; bottom - Stanton number.

grain roughness is $2.25 \mathrm{~mm}$, the corrected value of the wetted area ratio 1.08 is used, and the roughness height is $1.09 \mathrm{~mm}$. This time the reduced value of $R e_{s}$ is about 400 . Again, SU2 results agree with experimental and literature results (see the bottom of Figure7). The turbulent Prandtl number correction improves the numerical prediction of the Stanton number.

\subsubsection{Validation of the 2PP correction}

The $2 \mathrm{PP}$ correction model is built to give results similar to the wall temperature shift equation (23). The experimental results, exploited by Ligrani et al. (1979) to calibrate the equation (23), are therefore used to validate the $2 \mathrm{PP}$ correction model. According 

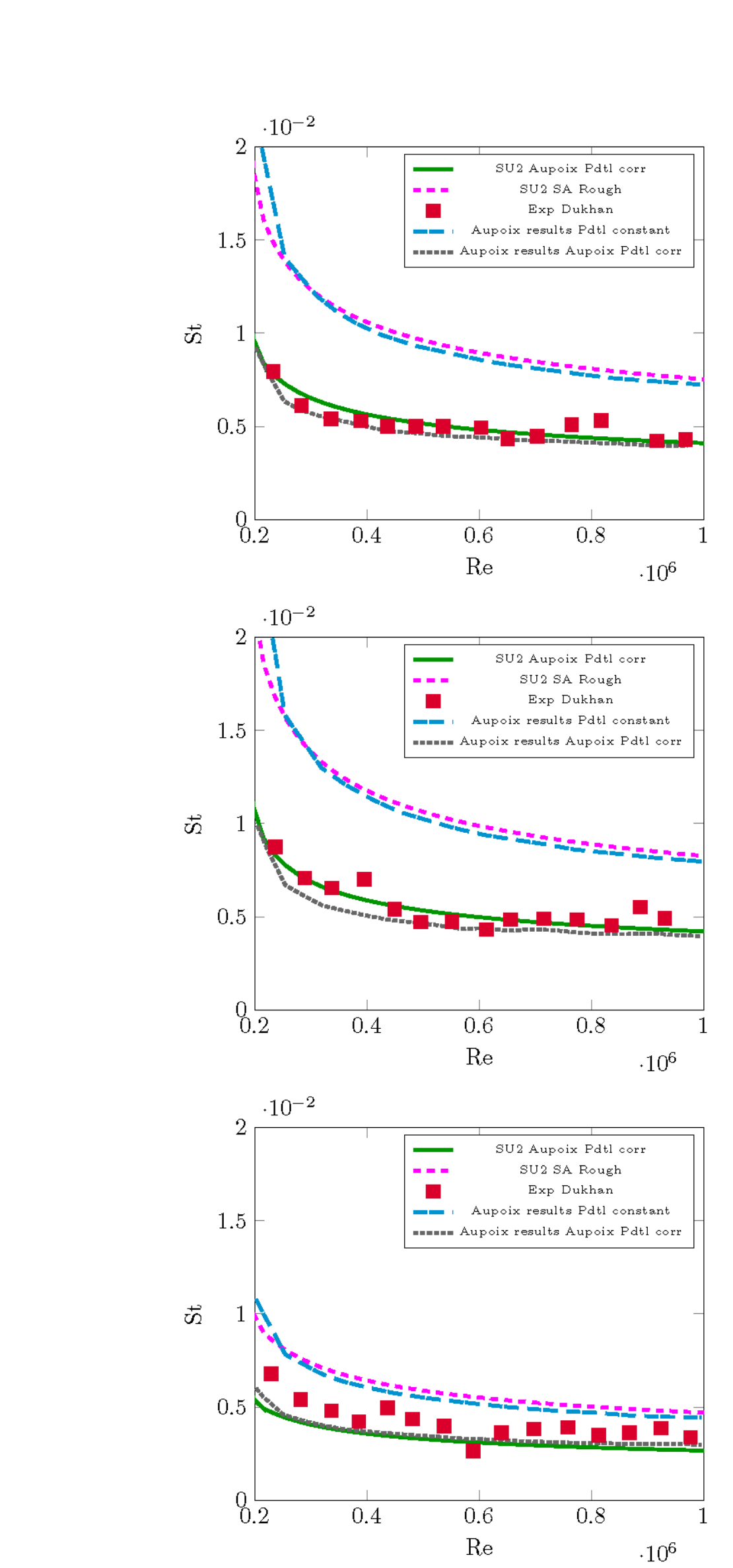

Figure 7. Dukhan et al. experiments (Aupoix 2015) - Stanton number distribution: top - intermediately spaced rough glaze; middle - rime feathers; bottom - smooth rime.

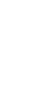


to Ligrani et al. or Healzer et al. (1974), the constant values for $\delta T_{0}^{+}$were selected such that:

$$
\delta T_{0}^{+}=\operatorname{Re}_{s}^{0.20} \operatorname{Pr}^{0.44}
$$

Ligrani et al. obtained experimental heat flux for flow over a flat plate covered with spheres, identical to that of Healzer et al. At the time, the equation (25) was implemented in a boundary layer method. The turbulent Prandtl number model was a function of the eddy diffusivity for momentum, as opposed to the constant $P r_{t}=0.9$ used with the RANS equations. A mixing length model was used for the turbulence model. The code was able to predict the experimental values well, but because of the differences in the mathematical model and in the numerical method, CFD results are not comparable. Thus, the CFD computed St are only compared to the experimental values.

For this test case only, the constant values for equation(34) are $C=1.0, \alpha=0.20$ and $\beta=0.44$. However, the results sensitivity to the constant choice is studied. The figure 8 shows that for the constant values $C=0.8$ or $C=1.0$, with $\alpha=0.20$ and $\beta=0.44$, the predicted Stanton numbers are almost identical. For the default values $C=1.92$, $\alpha=0.45$, and $\beta=0.8$, the Stanton is at most $2.5 \%$ lower. For this test case, the heat transfer is not particularly sensitive to the constant choice.

The SU2 results were obtained on the previously presented fine mesh, $200 \times 288$ grid nodes. The Stanton numbers for two velocities, $27.1 \mathrm{~m} / \mathrm{s}$ and $42.397 \mathrm{~m} / \mathrm{s}$, are shown in Figure 9. The maximum $y+$ are, for each velocity, $y+=0.3$ and $y+=0.45$. The results for the $2 \mathrm{PP}$ correction and the Aupoix Prandtl correction are both shown on the figure. The 2PP correction slightly underestimates the Stanton values, whereas the Aupoix Prandtl correction slightly overestimates the values. The difference between the $2 \mathrm{PP}$ correction and the experimental St is comparable to that between the numerical and experimental results shown in Ligrani et al. (1979). At the end of the plate, the Aupoix Prandtl correction $S t$ is $3 \%$ higher than $2 \mathrm{PP}$ correction prediction. If the default values for the constants model are used, the discrepancy increases to $5 \%$.

The Dukhan et al. (1999) experiments used for the Aupoix correction validations are again exploited here and a mesh sensitivity study for the $2 \mathrm{PP}$ model was conducted on the first case of Dukhan (the intermediately space rough glaze case of section 2.4.2). Three meshes were employed: i) coarse; $50 \times 77$, $\max y^{+}=1.95$, ii) medium; $100 \times 144$, $\max y^{+}=1.19$, and iii) fine; $200 \times 288, \max y^{+}=0.89$. The figure 10 shows that the results for the friction coefficient, $c_{f}$, and the Stanton, St, number tends toward similar values as the grid is refined. For the comparison between the two models, in the next section, the fine mesh is used.

\section{Comparison between Aupoix Prandtl correction and 2PP correction}

The heat transfers predicted by the two models are compared on flat plates, on a wing geometry, and on iced airfoils. For various icing conditions, the goal is to verify that the 2PP correction predicts heat transfer similar to the Aupoix Prandtl correction. Discrepancies between models are expected and will be quantified, as well as the discrepancy with the SA Rough model. 


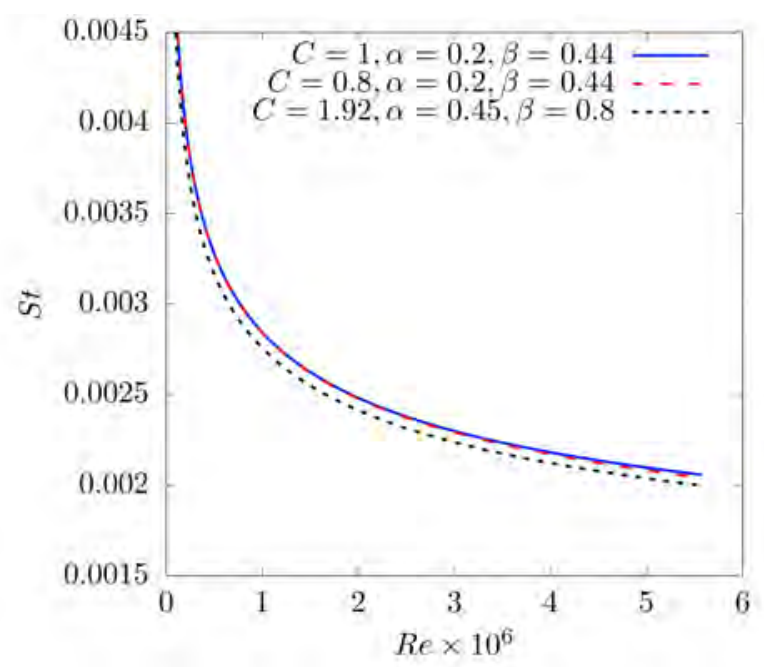

Figure 8 . Sensitivity study of the $2 \mathrm{PP}$ results to the $C, \alpha$, and $\beta$ constants

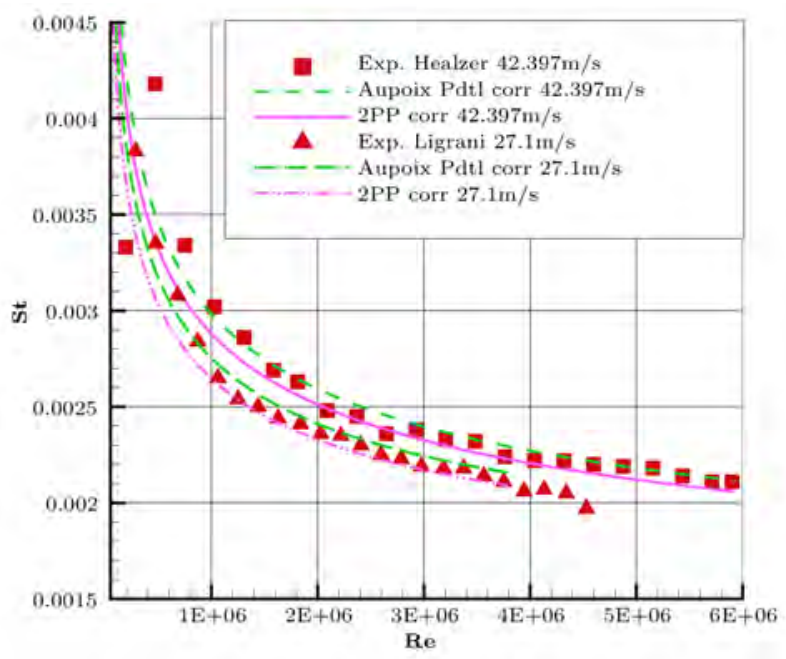

Figure 9. Healzer et al. (1974) and Ligrani et al. (1979) experiments, most closely packed spheres, SU2 Stanton number predictions with the two turbulent Prandtl correction models.

\subsection{Flat plates with ice accretion roughness}

Figure 11 presents a comparison between the two models for an intermediately spaced rough glaze ice, a roughness over rime feathers and a smooth rime ice case. Each plot shows five results. The experimental results and the no-roughness results are used as reference values. Three models for Stanton number predictions with SU2 are compared: the Aupoix Prandtl correction, the SA Rough model, and the 2PP correction model.

The two top graphs on figure 11 show the Stanton numbers for the intermediately spaced rough glaze ice case and the rime feathers case. The SA Rough model overesti- 

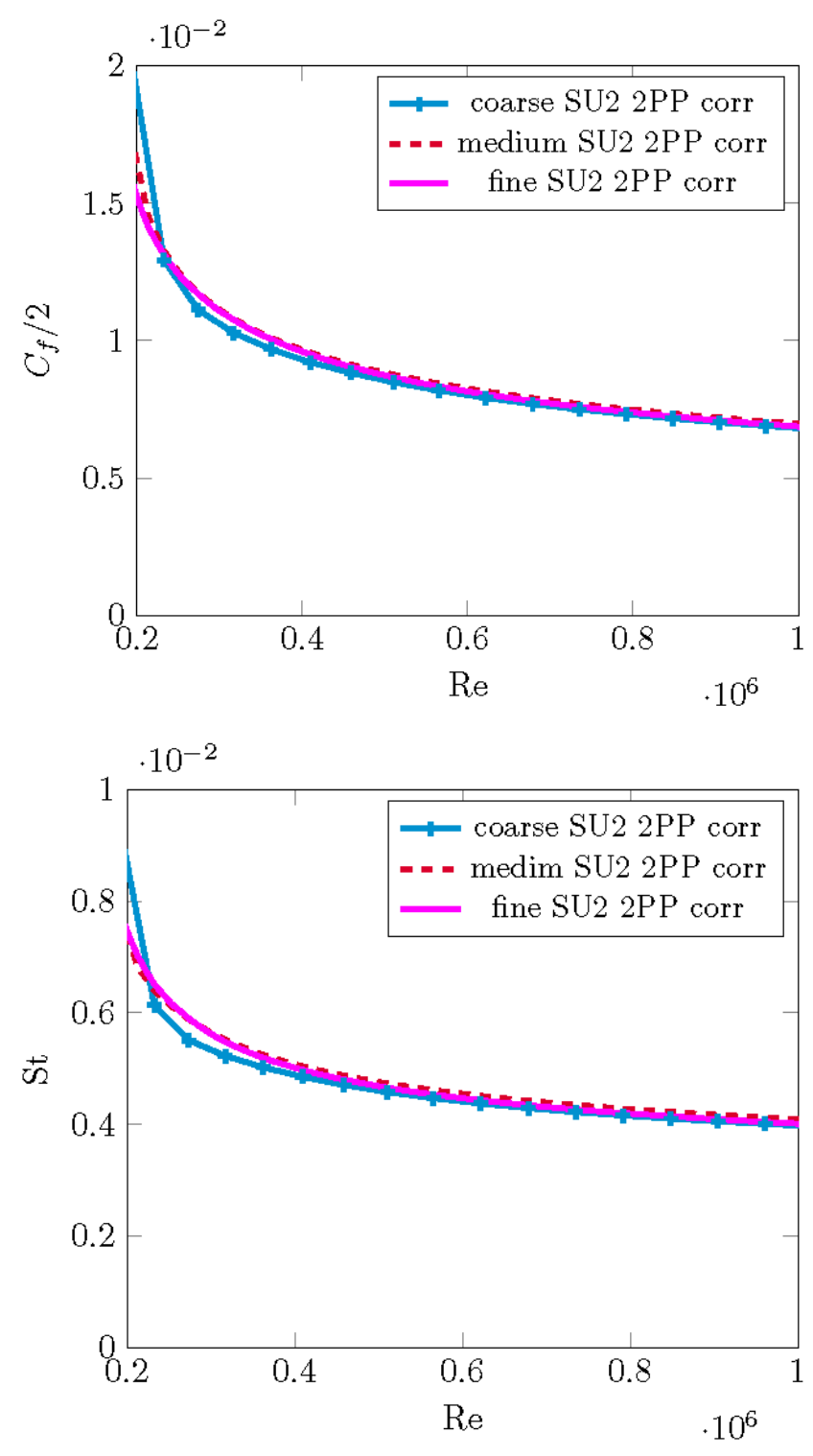

Figure 10. 2PP mesh sensitivity on Dukhan et al. - intermediately spaced rough glaze - top skin friction; - bottom: Stanton distribution.

mates by almost twice the experimental results. The two turbulent Prandtl correction models agree quite well with experiments, especially considering the clear experimental fluctuation with the Stanton number. For low Reynolds numbers, the Stanton number values predicted by the Aupoix Prandtl correction are around $20 \%$ higher than for the $2 \mathrm{PP}$ correction, but decrease faster with the Reynolds number. Thus, the values predicted by the Aupoix Prandtl correction get closer to the 2PP correction as the Reynolds number increases.

For the smooth rime case, bottom of Figure 11, the SA Rough model predictions are closer, but still higher than the experimental results. The discrepancy between the two turbulent Prandtl number correction models is around $15 \%$ and is almost independent of the Reynolds number. Contrary to the two top figures, the predictions for the Aupoix Prandtl correction model are lower than those of the 2PP. The results of the two models 
are reasonably close to experimental results, but the Aupoix Prandtl correction tends to underestimate the Stanton number.

\subsection{Wing based on NACA 4415 airfoil}

This test case illustrates the impact of the model choice on the heat transfer for a flow over a wing. The wing is based on a NACA 4415 airfoil, with a $30^{\circ}$ sweep, a $1 \mathrm{~m}$ chord, and an aspect ratio of 16 . This high aspect ratio reduces the importance of $3 D$ effects on the flow near the wing root. Our numerical set-up prescribed a free-stream temperature of $293 \mathrm{~K}$ and a heated temperature of $300 \mathrm{~K}$. In our simulations, the laminar/turbulent transition is not prescribed, and the test case is run fully turbulent. The free-stream velocity is set to $47.24 \mathrm{~m} . \mathrm{s}^{-1}$ at an angle of attack of $0^{\circ}$. Figure 12 shows the pressure coefficient on the wing surface, along with contours for $2 \mathrm{D}$ cut planes located at $y=$ 0,4 , and $7 \mathrm{~m}$. The computed eddy viscosity colors the symmetry plane and the plane located 10 chords downstream from the leading edge. An iso-surface for an eddy viscosity value of $0.01 \mathrm{~m}^{2} \mathrm{~s}^{-1}$ is plotted to help visualize the wake behind the wing.

The first $15 \%$ of the chord of the NACA 4415 wing is rough (Figure 13). The equivalent sand grain roughness used is $h_{s}=1 \mathrm{~mm}$, with a corrected wetted area ratio of $S_{c o r r}=$ 1.22 , and a roughness height of $h=0.1964 \mathrm{~mm}$. Three simulations were performed: one with the Aupoix Prandtl correction, one using the SA Rough model, and one using the 2PP correction.

On the left part of Figure 14, the friction coefficients in the rough region near the leading edge are shown as a function of the curvilinear distance, $s$, with $s=0$ at stagnation point. The coefficients are shown along a $2 \mathrm{D}$ cut at $y=4 \mathrm{~m}$ as an example, but a similar agreement between results is observed along the wingspan. The $R e_{s}$ values range from around 200 at the leading edge to a maximum value of 450 around $s / c \pm 0.05$. The turbulent Prandtl number correction models change the heat transfer near the wall and in the thermal boundary layer, but not the momentum boundary layer. The minor changes in friction coefficient are caused by the dynamic viscosity variations. The air viscosity changes with the temperature according to the Sutherland law.

Conversely, the Stanton number are reduced by $\approx 20 \%$ by the turbulent Prandtl correction models when compared to the SA Rough model. As expected, both Aupoix and 2PP correction models reduce the Stanton number near the leading edge of the wing (right part of Figure 14). Because the discrepancies between the model predictions are almost constant along the span, only the St at $y=4 m$ are shown as a function of the curvilinear distance. At the stagnation point, near $s / c=0$, the Stanton number has a local minimum and then starts to increase to reach maximum values near $s / c= \pm 0.02$. The sweep angle creates a secondary flow on the wing in the spanwise direction, but both correction models behave similarly and have less than $3 \%$ of discrepancy. Compared to the SA Rough model, the turbulent Prandtl number correction model changes the distribution shape only slightly, and mostly reduce the magnitude of the St. For this case, the 2PP correction model reduces the heat transfer slightly more than Aupoix's correction.

\section{3. $\quad$ Iced airfoil}

The thermal models are intended to predict heat transfer above iced surfaces. Therefore, the model predictions will be compared for flow over ice shapes typical of airfoil glaze ice accretions, as glaze ice shapes are more sensitive to heat transfer predictions. The two glaze ice geometries are well documented in the literature (AG-32 2003). Compared to 

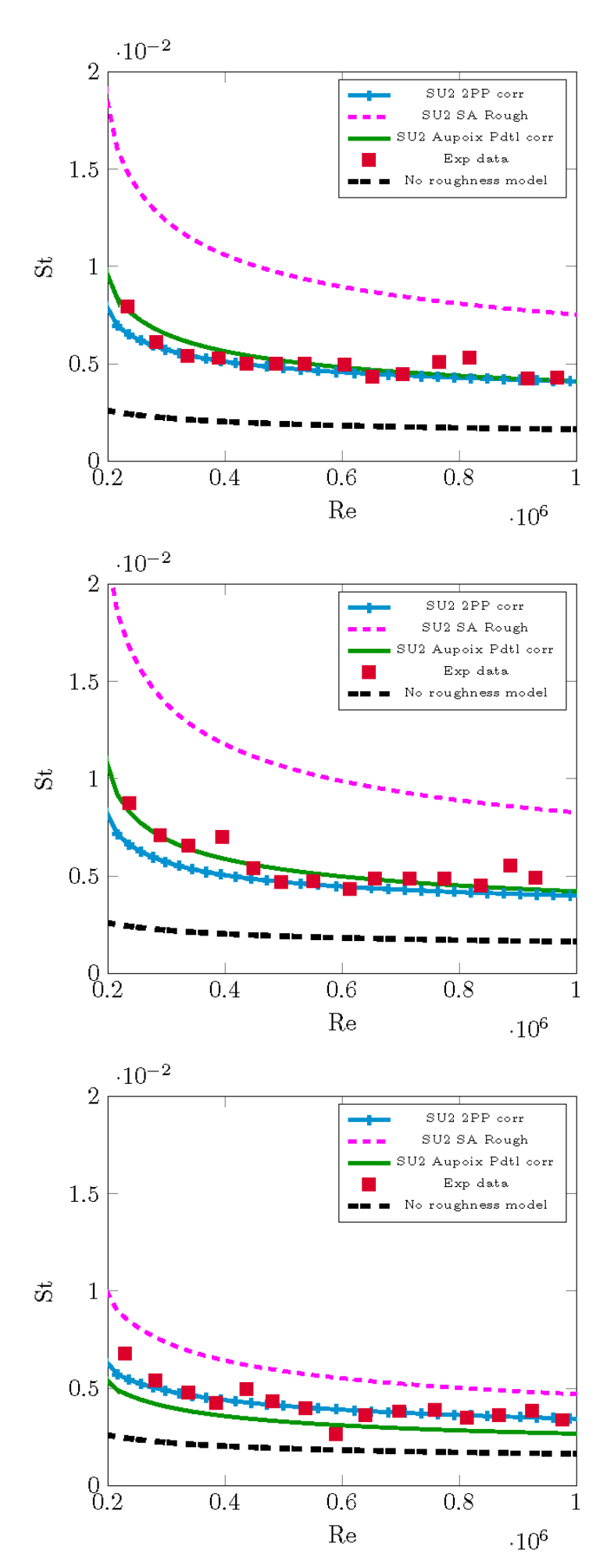

Figure 11. Dukhan et al. experiments - Stanton number distribution: top - intermediately spaced rough glaze; middle - rime feathers; - bottom: smooth rime.

(1)

International Journal of Computational Fhuid Dymaics

(1) 


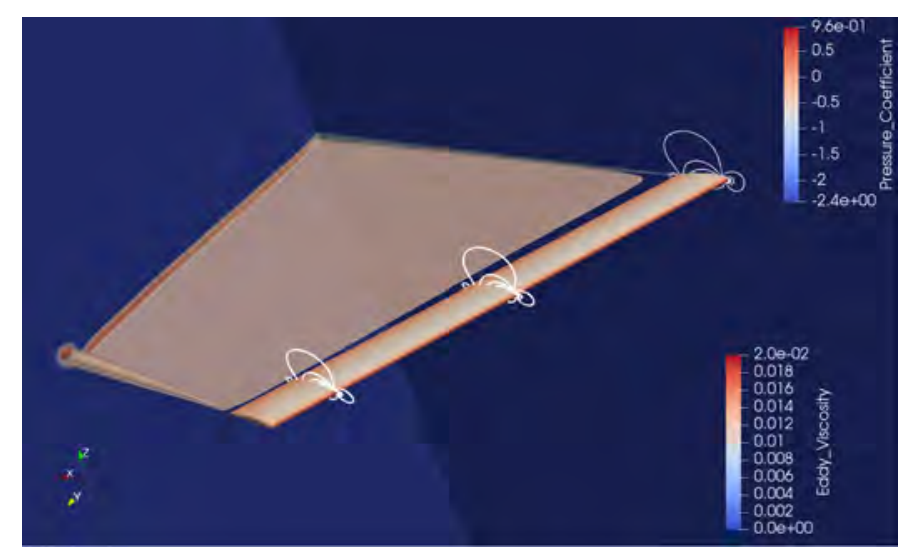

Figure $12.30^{\circ}$ sweep NACA4415 wing: pressure coefficient on the wing and eddy viscosity in the flow.

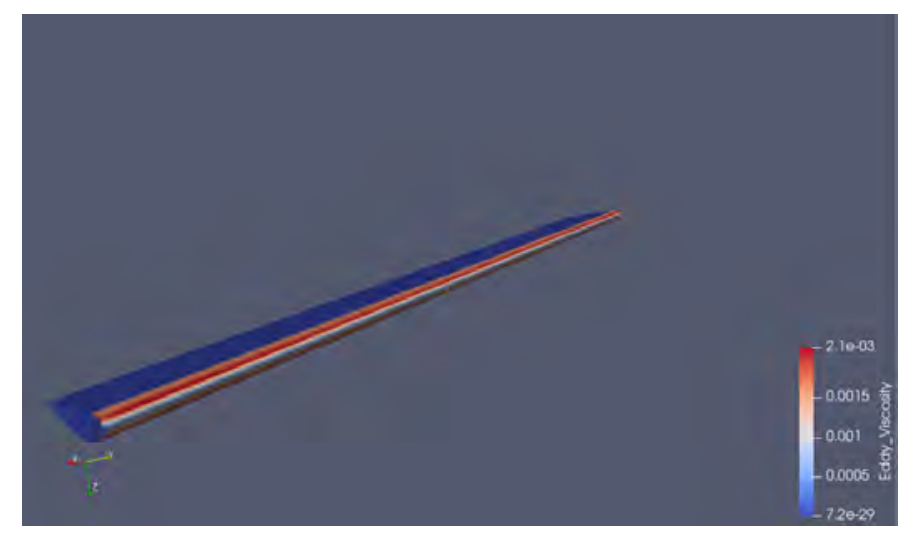

Figure 13. NACA4415 swept wing, turbulent eddy viscosity distribution on the wing.

the previous results, ice accretions create flows with steep changes in pressure gradient. This leads to large variations of the roughness Reynolds number along the iced surface.

\subsubsection{Iced NACA 0012}

The test case B from Garteur report AG-32 (2003) consists of a glaze ice shape formed on a NACA0012 airfoil. The studied ice shape was originally obtained in the NASA Lewis Icing Research Tunnel at ground level pressure for an airfoil of chord $c=0.5334 \mathrm{~m}$ at an angle of attack of $4^{\circ}$. The free-stream velocity was $58.1 \mathrm{~m} / \mathrm{s}$, the temperature was $-7.8^{\circ} \mathrm{C}$, the liquid water content $\mathrm{LWC}=2.1 \mathrm{~g} / \mathrm{m}^{3}$, the droplet diameter $20 \mu \mathrm{m}$, and the accretion time was $300 \mathrm{~s}$. This original ice shape was smoothed and manufactured for performance degradation study in a conventional wind tunnel Bragg (1986). Although the ice shape studied was smooth and thus assumed without roughness, the test case was selected because extensive experimental and numerical data are available. Because of the low wind speed tunnel and the smooth ice shape, the flow is probably laminar 


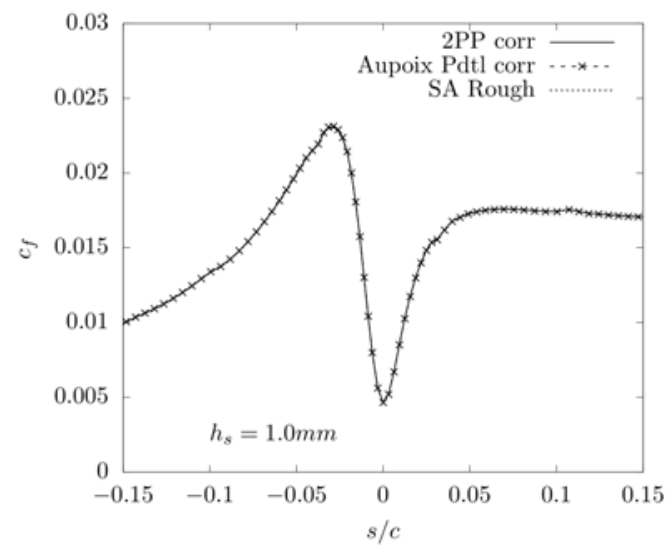

a)

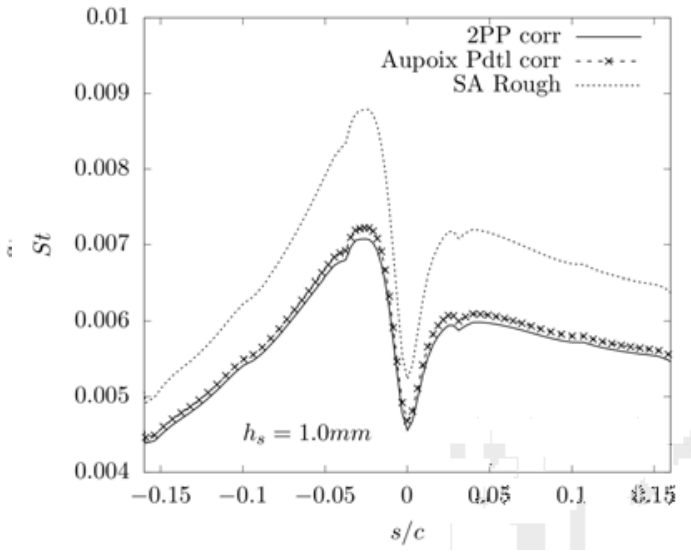

b)

Figure 14. NACA4415 swept wing, a) Friction coefficients along a $2 \mathrm{D}$ cut at $4 m$ in the spanwise direction; b) Stanton number along a $2 \mathrm{D}$ cut at $4 \mathrm{~m}$.

Table 1. Main grid properties

\begin{tabular}{cccc}
\hline grid & total nodes & surface nodes & $y_{\text {max }}^{+}$ \\
\hline coarse & 90349 & 218 & 0.25 \\
medium & 203504 & 326 & 0.18 \\
fine & 457284 & 489 & 0.11 \\
\hline
\end{tabular}

near the stagnation point in the experiment, however in the numerical simulation, a fully turbulent flow is assumed.

The test case B is used to compare the heat transfer predicted by the two models on a glaze ice accretion obtained at a relatively low airspeed. Even if the smooth glaze ice shape model was initially built without roughness, three roughness conditions representative of glaze ice conditions are applied for CFD simulations. Three C-grids are generated around the airfoil, extending $\approx 50$ chords away from the airfoil leading edge. The topology of the coarse grid near the airfoil is depicted in Figure 15. The table 1 presents the total number of nodes, the number of nodes positioned on the iced airfoil surface and the maximum $y^{+}$for each grid. The growth ratio between the grids is 1.5 in each direction. The $y_{\max }^{+}$ values are obtained from the CFD solutions. The element size in the normal direction is smaller near the surface, with the first node positioned around $2 \times 10^{-6} \mathrm{~m}$ above the surface for the medium grid.

Reference results, both numerical and experimental, are available for the smooth iced airfoil at $\operatorname{Re}=1.5 \times 10^{6}$ and $M a=0.12$. The pressure distribution for $\alpha=4^{\circ}$ is used to verify the CFD predicted flow field coherence above smooth surface. The reference results were obtained with an adiabatic iced airfoil. But in this article, the airfoil surface temperature is imposed to $308 \mathrm{~K}$, and the free stream temperature to $264.5 \mathrm{~K}$, in order to obtain heat fluxes. The Figure 16 shows a close-up view on the iced leading edge of the airfoil. The surface of interest, the area between $s / c=0$ and $s / c=0.1076$ is highlighted. 


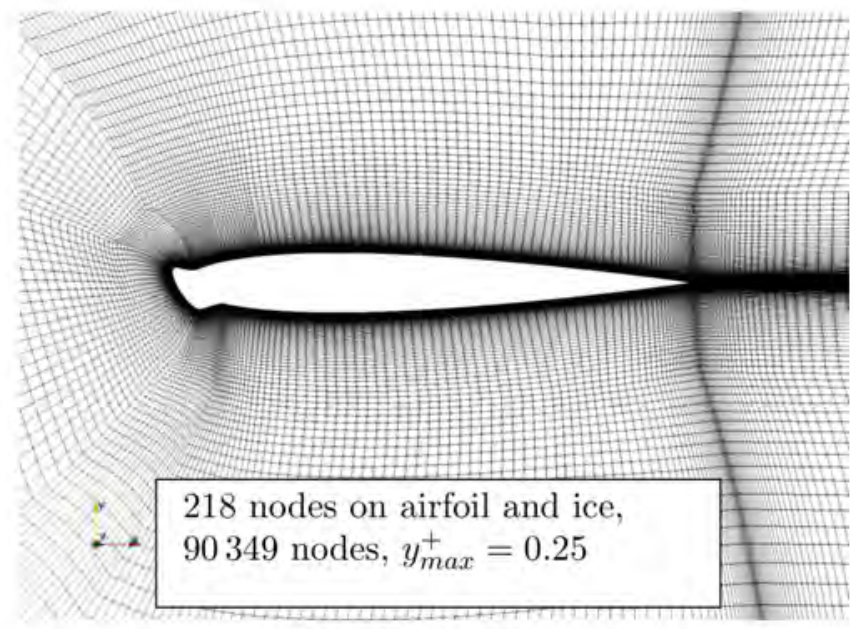

Figure 15. Coarse grid around the iced NACA 0012

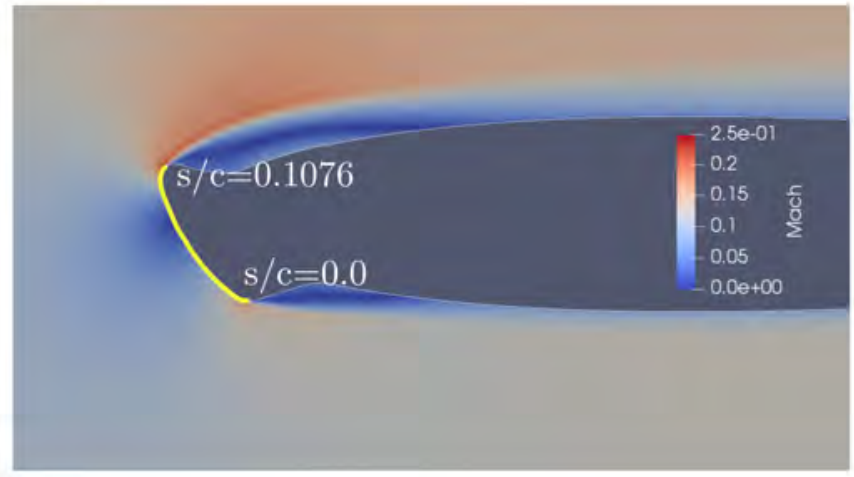

Figure 16. Mach iso-contours around the smooth ice shape. Studied surface is highlighted.

The curvilinear distance starting point is associated with a geometric feature of the ice shape, the lower horn. The Mach number iso-contours indicate the presence of separation areas on the upper surface and on the lower surface of the ice shape. The figure also shows the Mach number increases to 0.25 at the edge of the frontal part of the ice.

The computed pressure coefficients are compared to both experimental and numerical results (AG-32 2003). It is to be noticed that none of the CFD code pressure coefficients available in the literature (Dezitter et al. 2009) agree with the experiments in the separation regions. The figure 17 shows the $C_{p}$ computed on the three meshes with SU2 and without roughness model. The $C_{p}$ evolves slightly with the grid in the separation regions, from $x / c=0.05$ to $x / c=0.15$, with the fine grid results closer to the Fluent SA reference numerical results. The results in the flow separation regions are sensitive to the surface grid distribution near the separation points. Outside of the separation regions, the predicted pressure coefficients agree with the experimental results. On the right, the 

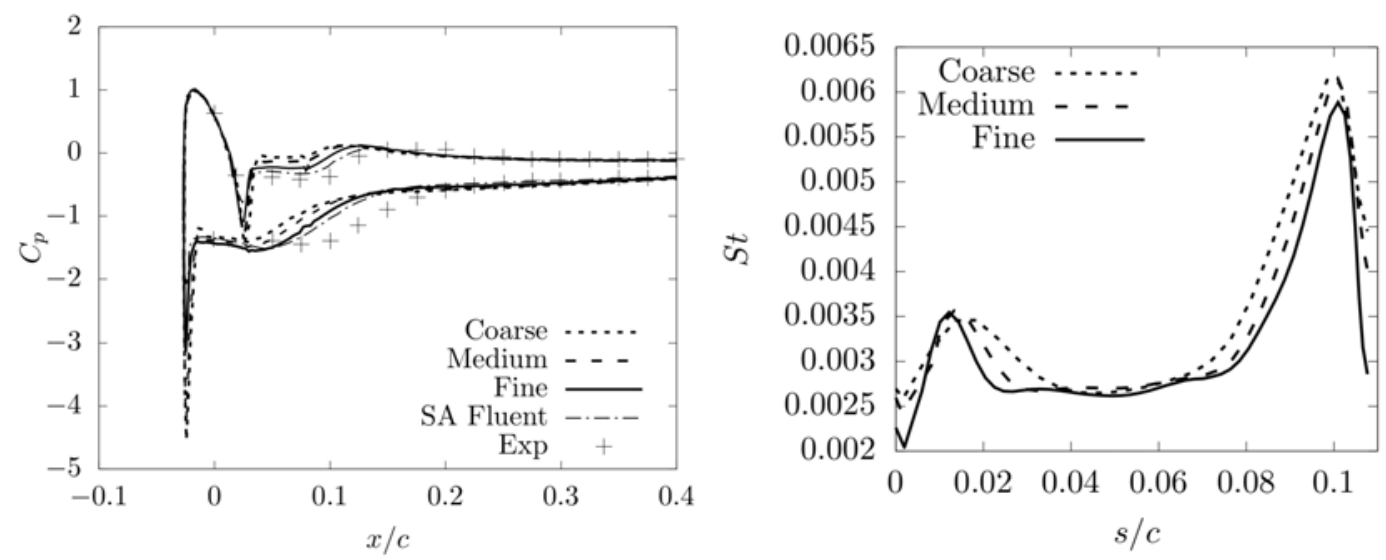

Figure 17. Left: pressure coefficient around iced NACA 0012 comparison between SU2 results on three grids, Fluent results and experimental results; and right: Stanton number on the surface of interest

Table 2. Iced airfoil with roughness test case.

\begin{tabular}{ccccc}
\hline Case & regime & $h_{s} / c \times 10^{3}$ & $S_{\text {corr }}$ & $h / h_{s}$ \\
\hline Model 2 & fully rough & 2.94 & 1.18 & 0.976 \\
Model 3 & mixed smooth transitional & 0.89 & 1.22 & 0.1964 \\
Model 6 & mixed smooth transitional & 1.00 & 1.06 & 10.25 \\
\hline
\end{tabular}

figure 17 shows the grid density effect on the Stanton number. The stagnation point is located around $s / c=0.07$. The minimum St values occur just upstream of the lower horn, near $s / c=0.05$, and at both separation points, near $s / c=0$ and $s / c=0.108$. The St reaches the maximum values just before the separation points. From coarse to medium grid, the maximum values have a $13 \%$ increase, and from medium to fine, $5 \%$. The separation point locations are displaced of about $1.7 \times 10^{-3} s / c$ from coarse to fine grid.

The ice shape corresponds to a smooth glaze model ice accretion, but no information is available about the roughness for the original ice accretion. The roughness parameters for Model 2, 3 and 6 of Dukhan test-cases, as defined by Radenac et al. (2018), are added to the numerical ice shape. The $h_{s}$ values, defined for flat plates, are adapted to the airfoil geometry. The table 2 presents the three cases and the corresponding three parameters needed by the Aupoix correction. The $2 \mathrm{PP}$ correction only used $h_{s} / c$ and $h / h_{s}$.

In table 2, the Model 2 corresponds to a fully rough loosely-spaced rough glaze ice and the Model 3 corresponds to a fully rough intermediately-spaced rough glaze ice shape. In Model 3, the $h_{s} / c$ parameter is selected to match an equivalent sand grain roughness of $0.6 \mathrm{~mm}$ used in AG-32 (2003) for the model 5-6 airfoil presented in the section 3.3.2. In the Model 6 , the $h_{s} / c$ parameter is obtained by using the baseline approach of IGLOO2D Radenac et al. (2018). In Figure 18 the Stanton number is computed using the SA Rough model, the Aupoix Prandtl correction and the $2 \mathrm{PP}$ correction. The $R e_{s}$ is also plotted 
on the figure, to show the variation in the flow regime along the curvilinear distance. The Model 2 has $\operatorname{Re}_{s}$ values above 100 almost everywhere in the studied area. The Model 3 and 6 have lower values, with values below 70 in the transitionnaly rough regime for approximately half of the studied area. As expected, the SA Rough model overestimates the Stanton number, especially at high $\mathrm{Re}_{s}$ values. When compared to Aupoix Prandtl correction, the maximum discrepancies with SA Rough is $55 \%$ for Model 2, $14 \%$ for Model 3 , and $31 \%$ for Model 6 . Both Prandtl corrections agree along the ice shape for the Model 2,3 , and 6 . The maximum discrepancies between those two Prandtl corrections are 5\% for Model 3 and 1\% for Model 6, with the Stanton numbers for the 2PP correction slightly lower than those obtained with Aupoix's correction. The 2PP correction overestimates the Stanton number by a maximum of $3 \%$ for Model 2 when compared to Aupoix's correction.

\subsubsection{Model 5-6}

The geometry, figure 19, used for the following test case is known as model 5-6. This model represents an outer wing section of a transport aircraft. This test case corresponds to case C1 in the AG-32 final report, documented in AG-32 (2003), Dezitter et al. (2009) and Tagawa et al. (2018). The leading edge glaze ice shape was calculated by Fokker, for which the reference icing conditions were at an angle of attack of $4^{\circ}$, an ambient pressure of 0.57 bar, a speed of $101.2 \mathrm{~m} / \mathrm{s}$, an outside temperature of $-14.7^{\circ} \mathrm{C}$, a water concentration of $1.96 \mathrm{~g} / \mathrm{m}^{3}$, a median volumetric droplet diameter of $20 \mu \mathrm{m}$, an accretion time of $148 \mathrm{~s}$, and a chord of $1.257 \mathrm{~m}$. The model used for the aerodynamic measurement model as a chord of $0.6759 \mathrm{~m}$, and the ice shape has an average grain size of $h_{s}=0.6 \mathrm{~mm}$ (Tagawa et al. 2018). The roughness parameters for the Prandtl corrections have been derived assuming an intermediately-spaced rough glaze ice shape, in concordance with Model 3 from Radenac et al. (2018), i.e $h / h_{s}=0.1964$ and $S_{\text {corr }}=1.22$. The aerodynamic parameters are the one used in the AG-32 final report, $M a=0.2, R e=3 \times 10^{6}$, and $0^{\circ}$ angle of attack. The left part of figure 19 presents the ice shape used and the portion of the chord where the Stanton is plotted. As a convention, the curvilinear distance start at $s=0 \mathrm{~m}$ at the bottom of the ice shape and reach $s=0.1585 \mathrm{~m}$ at the top of the ice shape. The Stanton distribution is computed using the SA Rough, the Aupoix Prandtl correction and the $2 \mathrm{PP}$ correction. As expected, the constant Prandtl assumption overestimates the Stanton by maximum value $32 \%$. The maximum discrepancy between both Prandtl corrections is around $12 \%$ near $s=5 \times 10^{2}$. The $2 \mathrm{PP}$ correction predicts a Stanton slightly higher than Aupoix's correction on the bottom part of the ice shape.

\section{Conclusion}

Two models were implemented in SU2 to correct the predicted heat flux over rough surfaces. The models were based on a corrected turbulent Prandtl number near the wall. The first model was developed and calibrated by Aupoix; the second one was based on a temperature shift model initially developed for the integral boundary layer prediction to predict heat transfer over a rough surface. By integrating the equation for the normalized temperature $T^{+}$, the model use the effective turbulent Prandtl number equation to obtain

a two parameters Prandtl correction model. The Aupoix Prandtl correction and the 2PP correction models were successfully implemented and validated against literature results. The turbulent Prandtl number correction models reduce the magnitude of the heat transfer, and results are closer to experiments. For most of the cases presented here, the discrepancies between the 2PP and the Aupoix Prandtl correction model are small, 

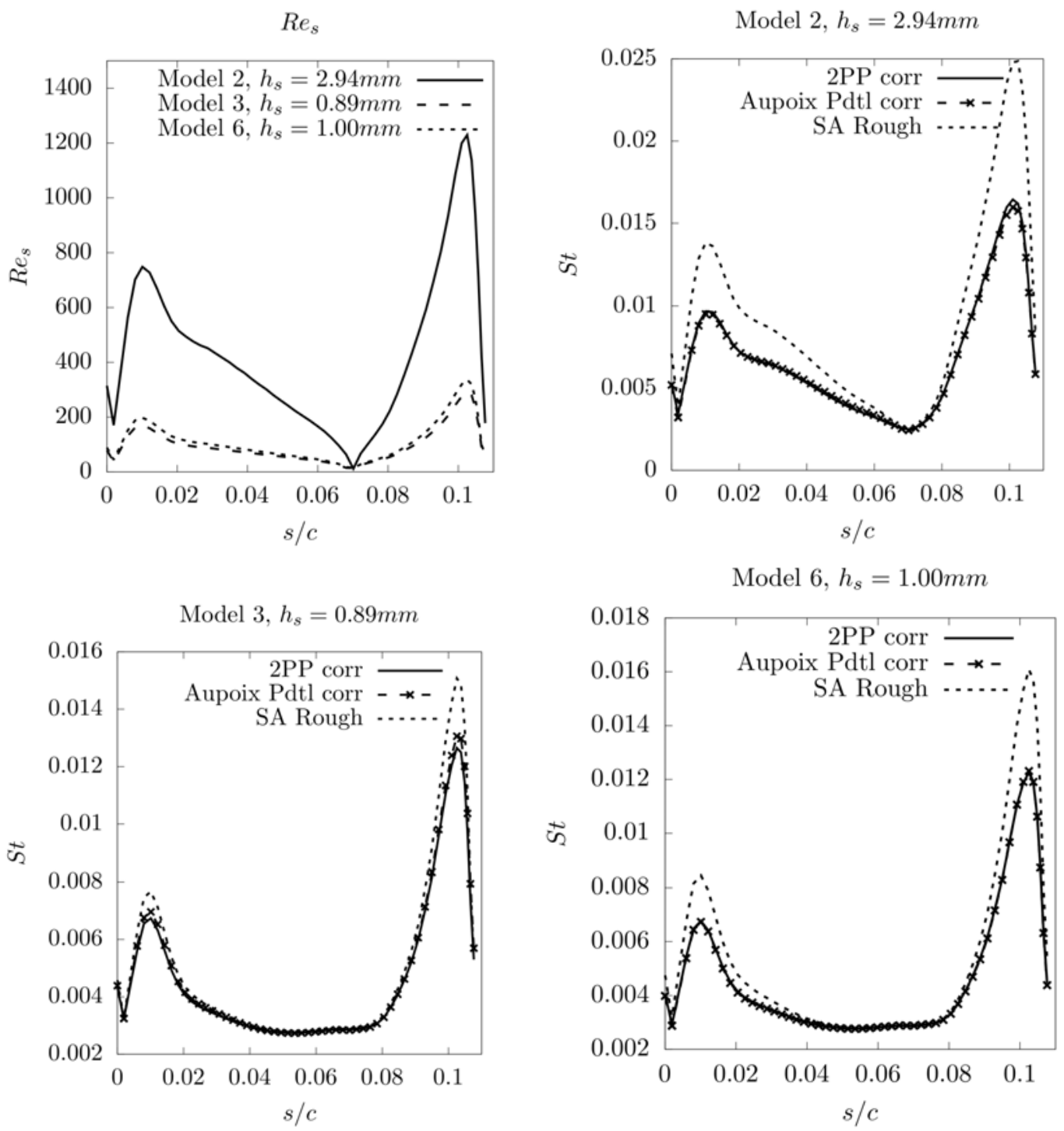

Figure 18. $\mathrm{Re}_{s}$ and St around a rough iced NACA0012, test case B from AG-32 (2003), comparisons between the SA Rough, the Aupoix Prandtl correction, and the 2PP correction

of the order of the typical experimental uncertainties on heat transfer measurements. In the worst cases, the discrepancy can reach a maximum of $12 \%$ for flow around an iced airfoil. Further work will consist in analyzing this effect on ice shape predictions.

\section{Acknowledgement}

The numerical experiments presented in this paper were carried in part out using MCIA (Mésocentre de Calcul Intensif Aquitain) facilities and the PLaFRIM experimental testbed. The PLaFRIM cluster is being developed under the Inria PLaFRIM development action with support from LABRI and IMB and other en- 

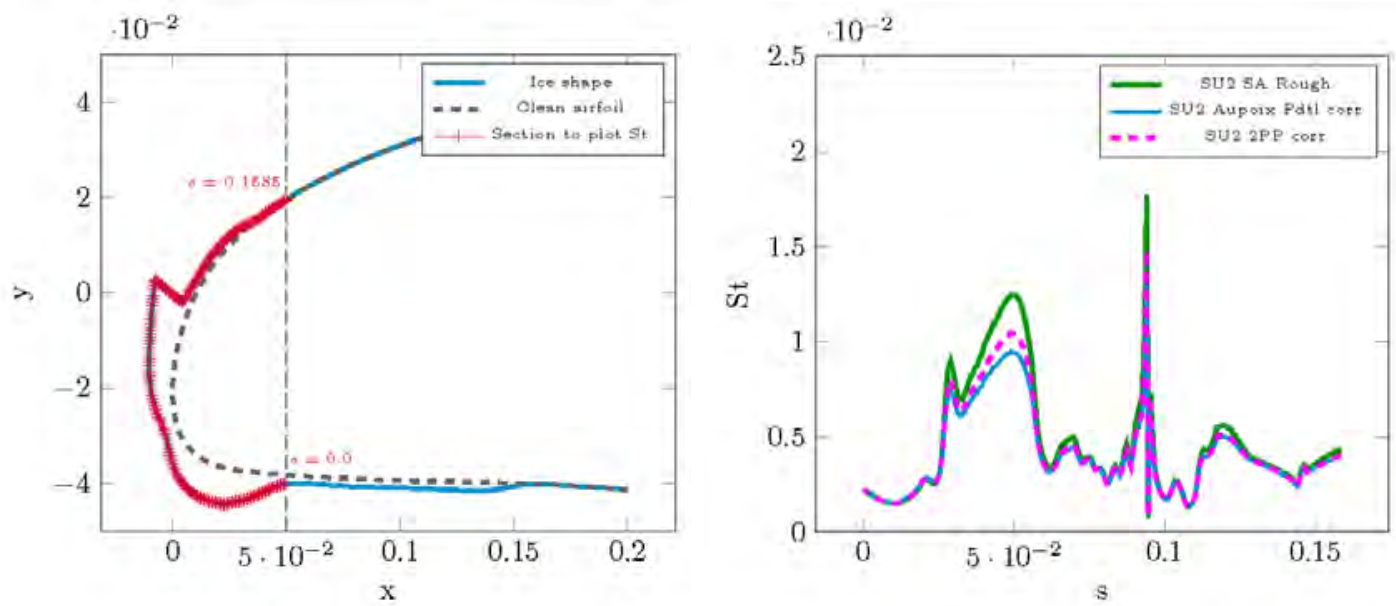

Figure 19. On the left: Model 5-6 setup, case C1 from AG-32 (2003): the portion of the chord where the Stanton is plotted is highlighted with symbols. On the right: Distribution of the Stanton along the chord for SA Rough, the Aupoix Prandtl correction and the 2PP correction.

tities: Conseil Régional d Aquitaine, FeDER, Université de Bordeaux and CNRS, see https://plafrim.bordeaux.inria.fr/. This research was enabled in part by the support provided by Calcul Québec (https://www.calculquebec.ca/) and Compute Canada (https://www.computecanada.ca/).

\section{Appendix A. Spalart-Allmaras Model with Wall Roughness Correction in SU2}

For the sake of completeness, we provide here the equations used for the wall roughness correction implementation in SU2 V5.2.

\section{A.1. Integral form of the Reynolds Averaged Navier-Stokes equations}

The integral form of the Navier-Stokes equation is written using convective fluxes, $\mathbf{F}_{\mathbf{c}}$, viscous fluxes, $\mathbf{F}_{\mathbf{v}}$, and conservative variables $\mathbf{W}$ in $\mathrm{SU} 2$ :

$$
\frac{\partial}{\partial t} \int_{\Omega} \mathbf{W} d \Omega+\oint_{\partial \Omega}\left(\mathbf{F}_{\mathbf{c}}-\mathbf{F}_{\mathbf{v}}\right) d S=0
$$

where

$$
\mathbf{W}=\left(\begin{array}{c}
\rho \\
\rho u_{1} \\
\rho u_{2} \\
\rho u_{3} \\
\rho E
\end{array}\right) \mathbf{F}_{\mathbf{c}}=\left(\begin{array}{c}
\rho V \\
\rho u_{1} V+n_{1} p \\
\rho u_{2} V+n_{2} p \\
\rho u_{3} V+n_{3} p \\
\rho H V
\end{array}\right) \mathbf{F}_{\mathbf{v}}=\left(\begin{array}{c}
0 \\
n_{i} \tau_{1 i} \\
n_{i} \tau_{2 i} \\
n_{i} \tau_{3 i} \\
n_{i} \Theta_{i}
\end{array}\right)
$$

with $V=n_{i} u_{i}, H=E+p / \rho$ and 


$$
\Theta_{i}=u_{j} \tau_{i j}+k \frac{\partial T}{\partial x_{i}}
$$

For aerodynamic flows, the air is considered as a perfect gas. The pressure $p$ and enthalpy $H$ are

$$
\begin{gathered}
p=\rho R T \\
H=c_{p} T
\end{gathered}
$$

Knowing that $R=c_{p}-c_{v}$ and $\gamma=c_{p} / c_{v}$, the pressure is expressed in term of conservative variables

$$
p=(\gamma-1) \rho\left(E-\frac{u_{i} u_{i}}{2}\right)
$$

The Sutherland formula is used to compute the dynamic viscosity $\mu_{L}$

$$
\mu_{L}=\frac{1.45 T^{3 / 2}}{T+110} \times 10^{-6}
$$

and the conductivity coefficient $k_{L}$ is linked to the viscosity through the Prandtl number

$$
k_{L}=c_{p} \frac{\mu_{L}}{\operatorname{Pr}}
$$

The fluid is assumed to be Newtonian, therefore the components of the viscous stress tensor are for the flow velocity $\mathbf{v}=\left(u_{1}, u_{2}, u_{3}\right)$

$$
\tau_{i j}=\mu\left(\frac{\partial u_{i}}{\partial x_{j}}+\frac{\partial u_{j}}{\partial x_{i}}-\frac{2}{3} \delta_{i j} \nabla \cdot \mathbf{v}\right)
$$

The eddy viscosity hypothesis of Boussinesq is used to model the turbulent shear stress. Herewith, the dynamic viscosity is replaced by the sum of the laminar and turbulent eddy viscosity in the viscous stress tensor

$$
\mu=\mu_{L}+\mu_{t}
$$

Similarly, the viscous turbulent heat flux is obtained by replacing the conductivity coefficient by the sum of the laminar and turbulent thermal conductivity 


$$
k=k_{L}+k_{t}=c_{p}\left(\frac{\mu_{L}}{P r}+\frac{\mu_{t}}{P r_{t}}\right)
$$

The turbulent eddy viscosity has to be determined with the aid of a turbulence model.

\section{A.2. Wall Roughness Correction in Spalart-Allmaras One-Equation Model}

Several models are possible to compute the turbulent eddy viscosity. One popular model in aerodynamics is the Spalart-Allmaras (SA) model. Among the various forms of the model, we have implemented the standard SA model with a correction to account for wall roughness, as proposed by Boeing (Aupoix and Spalart 2003).

The one-equation model is given by, with $\nu=\mu_{L} / \rho$,

$$
\begin{aligned}
\frac{\partial \tilde{\nu}}{\partial t}+u_{j} \frac{\partial \tilde{\nu}}{\partial x_{j}}= & c_{b 1}\left(1-f_{t 2}\right) \hat{S} \tilde{\nu}-\left(c_{w 1} f_{w}-\frac{c_{b 1}}{\kappa^{2}} f_{t 2}\right)\left(\frac{\tilde{\nu}}{d}\right)^{2} \\
& +\frac{1}{\sigma}\left(\frac{\partial}{\partial x_{j}}\left((\nu+\tilde{\nu}) \frac{\partial \tilde{\nu}}{\partial x_{j}}\right)+c_{b 2} \frac{\partial \tilde{\nu}}{\partial x_{i}} \frac{\partial \tilde{\nu}}{\partial x_{i}}\right)
\end{aligned}
$$

and the turbulent eddy viscosity is computed

$$
\mu_{t}=\rho \tilde{\nu} f_{v 1}
$$

To account for roughness the distance to the nearest wall, $d$, is augmented to read $d_{\text {new }}=d+0.03 h_{s}$, with $h_{s}$ the equivalent sand grain roughness. The definition of $\chi$ is also modified such that

$$
f_{v 1}=\frac{\chi^{3}}{\chi^{3}+c_{v 1}^{3}} \quad \chi=\frac{\tilde{\nu}}{\nu}+c_{R 1} \frac{h_{s}}{d_{n e w}}
$$
$\hat{S}$

$$
\begin{array}{cc}
\hat{S}=\Omega+\frac{\tilde{\nu}}{\kappa^{2} d_{n e w}^{2}} f_{v 2} & \Omega=\sqrt{2 \omega_{i j} \omega_{i j}} \\
f_{v 2}=1-\frac{\tilde{\nu}}{\nu+\tilde{\nu} f_{v 1}} & f_{w}=g\left(\frac{1+c_{w 3}^{6}}{g^{6}+c_{w 3}^{6}}\right)^{1 / 6} \\
g=r+c_{w 2}\left(r^{6}-r\right) & r=\min \left(\frac{\tilde{\nu}}{\hat{S} \kappa^{2} d_{n e w}^{2}}, 10\right) \\
f_{t 2}=c_{t 3} \exp \left(-c_{t 4} \chi^{2}\right) & \omega_{i j}=\frac{1}{2}\left(\frac{\partial u_{i}}{\partial x_{j}}-\frac{\partial u_{j}}{\partial x_{i}}\right)
\end{array}
$$

and the constants are 


$$
\begin{array}{ccc}
c_{b 1}=0.1355 & \sigma=2 / 3 & c_{b 2}=0.622 \\
\kappa=0.41 & c_{w 2}=0.3 & c_{w 3}=2 \\
c_{v 1}=7.1 & c_{t 3}=1.2 & c_{t 4}=0.5 \\
c_{w 2}=\frac{c_{b 1}}{\kappa^{2}}+\frac{1+c_{b 2}}{\sigma} & c_{R 1}=0.5 &
\end{array}
$$

Most importantly, the wall boundary condition is not $\tilde{\nu}_{w}=0$ as in the original model, but

$$
\left(\frac{\partial \tilde{\nu}}{\partial n}\right)_{w}=\frac{\tilde{\nu}_{w}}{0.03 h_{s}}
$$

with $n$ along the wall normal.

\section{References}

AG-32, G.A.G., Prediction of performance degradation due to icing for 2D configurations. , 2003. , Report, GARTEUR Action Group AG-32.

Anderson, D.N., Hentschel, D.B., and Ruff, G.A., 2003. Measurement and Correlation of Ice Accretion Roughness. In: AIAA-98-0486 https://doi.org/10.2514/6.1998-486.

Anderson, D.N. and Shin, J., 1997. Characterization of Ice Roughness From Simulated Icing Encounters. In: AIAA-97-0052 https://doi.org/10.2514/6.1997-52, 35th Aerospace Sciences Meeting and Exhibit, Reno, Nevada.

Aupoix, B., 2007. A General Strategy to Extend Turbulence Models to Rough Surfaces: Application to Smiths k-L Model. Journal of Fluids Engineering, 129 (10), 1245-1254 http://dx.doi.org/10.1115/1.2776960.

Aupoix, B., Couches limites bidimensionnelles compressibles. Descriptif et mode demploi du code CLICET version 2010. , 2010. , Report Tech. Rep. RT 1/117015 DMA, ONERA.

Aupoix, B., 2015. Improved heat transfer predictions on rough surfaces. International Journal of Heat and Fluid Flow, 56, 160-171 http://dx.doi.org/10.1016/j.ijheatfluidflow.2015.07.007.

Aupoix, B. and Spalart, P.R., 2003. Extensions of the Spalart-Allmaras Turbulence Model to Account for Wall Roughness. International Journal of Heat and Fluid Flow, 24, 454462 http://dx.doi.org/10.1016/S0142-727X(03)00043-2.

Beaugendre, H., Morency, F., and Habashi, W.G., 2003. FENSAP-ICERoughness effects on ice shape prediction. In: AIAA-Paper 2003-1222, January. https://doi.org/10.2514/6.2003-1222, 41st Aerospace Sciences Meeting and Exhibit, Reno, Nevada.

Beaugendre, H., Morency, F., and Habashi, W.G., 2006. Development of a Second Generation In-Flight Icing Simulation Code. Journal of Fluids Engineering, Transactions of the ASME, 128 (2), 378-387 https://doi.org/10.1115/1.2169807.

Blazek, J., 2015. Computational Fluid Dynamics: Principles and Applications. Third Edition Elsevier Ltd.

Bragg, M.B., An Experimental Study of the Aerodynamics of a NACA 0012 Airfoil with a Simulated Glaze Ice Accretion. , 1986. , Report NASA-CR-179897, NASA. 
Calvo, J.B., 2009. Numerical Simulation of Liquid Rocket Engine Cooling Channels. Thesis $(\mathrm{PhD})$. Departament de Mecànica de Fluids, Universitat Politècnica de Catalunya (UPC) Deutsches Luftund Raumfahrt Zentrum (DLR).

Chedevergne, F., 2018. Analytical wall function including roughness corrections. International Journal of Heat and Fluid Flow, 73, 258-269 https://doi.org/10.1016/j.ijheatfluidflow.2018.08.001.

Corson, D., Jaiman, R., and Shakib, F., 2009. Industrial application of RANS modelling: capabilities and needs. International Journal of Computational Fluid Dynamics, 23 (4), 337-347 https://doi.org/10.1080/10618560902776810.

Dezitter, F., et al., 2009. Enhancement of Prediction Capability in Icing Accretion and Related Performance Penalties Part II: CFD Prediction of the Performance Degradation due to Ice. In: 1st AIAA Atmospheric and Space Environments Conference,San Antonio, Texas, June. https://doi.org/10.2514/6.2009-3970, American Institute of Aeronautics and Astronautics.

Dipprey, D.F. and Sabersky, R.H., 1963. Heat and Momentum Transfer in Smooth and Rough Tubes at Various Prandtl Numbers. Int J. Heat Mass Transfer, 6, 329-335 http://dx.doi.org/10.1016/0017-9310(63)90097-8.

Dirling, Jr., R.B., 1973. A method for computing roughwall heat transfer rates on reentry nosetips. In: AIAA Paper 73-763, July 16. https://doi.org/10.2514/6.1973-763, AIAA 8th Thermophysics Conference, Palm Springs, CA.

Dukhan, N., et al., 1999. Experimental heat transfer coefficients from iceroughened surfaces for air deicing design.. Journal of Aircraft, 36 (6), 948-956 https://doi.org/10.2514/2.2556.

Economon, T.D., et al., 2016. SU2: An open-source suite for multiphysics simulation and design. AIAA Journal, 54 (3), 828-846 http://dx.doi.org/10.2514/1.J053813.

Han, Y. and Palacios, J., 2017. Surface Roughness and Heat Transfer Improved Predictions for Aircraft Ice-Accretion Modeling. AIAA Journal, 1-14 https://doi.org/10.2514/1.J055217.

Hanson, D.R. and Kinsel, M.P., 2019. Evaluation of a Subgrid-Scale Computational Fluid Dynamics Model for Ice Roughness. Journal of Aircraft, 56 (2), 787-799 https://doi.org/10.2514/1.C035060.

Healzer, J.M., Moffat, R.J., and Kays, W.M., The Turbulent Boundary Layer on a Rough Porous Plate: Experimental Heat Transfer with Uniform Blowing. , 1974. , Report HMT-18, Stanford University.

Hink, R., Hannemann, V., and Eggers, T., 2014. Extension of the Spalart-Allmaras OneEquation Turbulence Model for Effusive Cooled Rocket Chambers. In: 4th International Space Propulsion Conference, May. https://elib.dlr.de/89895/.

Hosni, M.H., et al., 1993. Roughness element shape effects on heat transfer and skin friction in rough-wall turbulent boundary layers. International Journal of Heat and Mass Transfer, 36 (1), 147-153 https://doi.org/10.1016/0017-9310(93)80074-5.

Kays, W.M. and Crawford, M.E., 1993. Convective heat and mass transfer. 3rd McGrawHill series in mechanical engineering New York: McGraw-Hill.

Knopp, T., Eisfeld, B., and Calvo, J.B., 2009. A new extension for k $\omega$ turbulence models to account for wall roughness. International Journal of Heat and Fluid Flow, 30, 54-65 https://doi.org/10.1016/j.ijheatfluidflow.2008.09.009.

Ligrani, P.M., Moffat, R.J., and Kays, W.M., The thermal and hydrodynamic behavior of thick, rough-wall, turbulent boundary layers. , 1979. , Report HMT-29, Stanford University.

Liu, Y. and $\mathrm{Hu}, \mathrm{H} ., 2018$. An experimental investigation on the unsteady heat transfer 
process over an ice accreting airfoil surface. International Journal of Heat and Mass Transfer, 122, 707-718 https://doi.org/10.1016/j.ijheatmasstransfer.2018.02.023.

Owen, P. and Thomson, W., 1963. Heat transfer across rough surfaces. Journal of Fluid Mechanics, 15, 321-334 https://doi.org/10.1017/S0022112063000288.

Pailhas, G., Touvet, Y., and Aupoix, B., 2008. Effects of Reynolds number and adverse pressure gradient on a turbulent boundary layer developing on a rough surface. Journal of Turbulence, 9, N43 https://doi.org/10.1080/14685240802562020.

Radenac, E., et al., 2018. An extended rough-wall model for an integral boundary layer model intended for ice accretion calculations. In: AIAA 2018-2858 https://doi.org/10.2514/6.2018-2858, 2018 Atmospheric and Space Environments Conference, Atlanta, Georgia.

Rumsey, C.L., 2014. Turbulence Modeling Verification and Validation. In: AIAA 20140201, Jan. https://doi.org/10.2514/6.2014-0201, 52nd AIAA Aerospace Sciences Meeting,National Harbor, Maryland.

Shin, J., 1996. Characteristics of Surface Roughness Associated with Leading-Edge Ice Accretion. Journal of Aircraft, 33 (2), 316-321 https://doi.org/10.2514/3.46940.

Suga, K., Craft, T.J., and Iacovides, H., 2006. An analytical wall-function for turbulent flows and heat transfer over rough walls. International Journal of Heat and Fluid Flow, 27, 852-866 https://doi.org/10.1016/j.ijheatfluidflow.2006.03.011.

Tagawa, G.B., Morency, F., and Beaugendre, H., 2018. CFD study of airfoil lift reduction caused by ice roughness. In: AIAA Paper 2018-3010, June. https://doi.org/10.2514/6.2018-3010, 2018 Applied Aerodynamics Conference, Atlanta, Georgia.

Trontin, P., et al., 2017. Description and assessment of the new ONERA 2D icing suite IGLOO2D. In: 9th AIAA Atmospheric and Space Environments Conference, AIAA AVIATION Forum, Denver, Colorado, June. https://doi.org/10.2514/6.20173417, American Institute of Aeronautics and Astronautics.

Walker, C.W., McClain, S.T., and Shannon, T.A., 2014. Convection from Ice Roughness with Varying Flux Boundary Conditions. In: AIAA 2014-2463, June. https://doi.org/10.2514/6.2014-2463, 6th AIAA Atmospheric and Space Environments Conference,Atlanta,GA.

Wright, W.B., User's Manual for LEWICE Version 3.2. , 2008. , Report NASA/CR-2008214255, NASA. 